\title{
LA GEOMETRIA DELLO SPAZIO ELETTORALE IN ITALIA
}

\author{
di Luca Ricolfi
}

\section{Unidimensionalità imperfetta o multidimensionalità emergente?}

Quante dimensioni sono necessarie per rappresentare le posizioni dei partiti italiani nello spazio elettorale? La classica rappresentazione dei partiti lungo un unico continuum, il cosiddetto asse sinistra-destra, è ancora attuale? E se non lo è, quali sono le dimensioni oggi pertinenti?

Negli ultimi cinquant'anni il problema della unidimensionalità dello spazio elettorale ha sempre avuto un certo rilievo perché, dopo gli studi pionieristici di Black, Arrow e Coombs sul rapporto fra preferenze individuali e regole di scelta sociale, l'assunto di unidimensionalità è diventato, per così dire, il certificato di garanzia dei sistemi democratici. Solo assumendo uno spazio elettorale unidimensionale l'adozione della regola della maggioranza non incappa nel «paradosso del voto» su cui per primi, verso la fine del '700, avevano attirato l'attenzione JeanCharles de Borda (1781) e il Marchese di Condorcet (1785).

Sono grato a Giuseppe Giampaglia e Alberto Marradi per le loro osservazioni a una precedente versione di questo saggio.

1 Tra la fine degli anni quaranta e i primissimi anni cinquanta Arrow formula il suo celebre «teorema di possibilità», con cui si dimostra l'impossibilità di costruire una funzione del benessere sociale che rispetti alcune condizioni molto naturali (Arrow 1951); fra tali condizioni vi era quella che nessun ordine di preferenza individuale sia escluso a priori, una condizione che viola evidentemente l'assunto di unidimensionalità. Un modo per aggirare il teorema di Arrow è di assumere che le preferenze individuali siano soggette a determinate restrizioni più o meno ragionevoli, come quella di unidimensionalità. È allo studio di tali restrizioni che Black e Coombs, l'uno indipendentemente dall'altro, hanno fornito un contributo decisivo tra la fine degli anni quaranta $\mathrm{e} i$ primi anni cinquanta (Black 1948a; 1948b; 1948c; Coombs 1950; 1952; 1954). Una ricostruzione dei nessi fra gli studi sette e ottocenteschi sui metodi di elezione e la moderna teoria della scelta sociale si trova nel capitolo 8 dell'edizione 1963 del libro di Arrow, Social Choice and Individual Values.

RIVISTA ITALIANA DI SCIENZA POLITICA / a. XXIII, n. 3, dicembre 1993 
A queste ragioni di interesse per il problema dell'unidimensionalità se ne sono venute aggiungendo, negli ultimi decenni, almeno altre tre. La prima è che, dopo il fondamentale contributo di Downs all'analisi «economica» dei sistemi politici democratici (Downs 1957), l'assunto di unidimensionalità è divenuto uno dei tratti distintivi dei modelli della competizione partitica $^{2}$. Se l'assunto di unidimensionalità non tenesse, molto di quanto si è pensato e scritto sulla dinamica dei sistemi partitici andrebbe riconsiderato attentamente.

La seconda è che l'emergere di nuove issue della competizione partitica (si pensi all'ambiente, o alla moralizzazione della vita politica) e di nuove forze politiche portatrici di tali issue rende problematico l'assunto di unidimensionalità (Bürklin 1985; Raschke 1991; Hösle 1991). Non è affatto detto che tutte le distinzioni e le differenze percepite come rilevanti dall'elettorato siano adeguatamente rappresentabili mediante un unico continuum, sia esso il grado di intervento dello stato nell'economia (Downs 1957), la posizione sull'asse sinistra-destra, o qualche nuova e diversa dimensione capace di strutturare gli orientamenti elettorali3.

La terza ragione di interesse riguarda direttamente l'Italia, e il ridisegno delle regole istituzionali ed elettorali attualmente in corso. Capire quante e quali sono le dimensioni dello spazio elettorale può essere rilevante per immaginare quale potrà essere la geografia politica della seconda repubblica, quali alleanze e quali linee di divisione è ragionevole aspettarsi per il futuro.

Nei lavori che si sono occupati del problema della dimensionalità dello spazio politico in Italia non è difficile individuare una curiosa bipartizione. Da una parte i contributi dei «politologi puri», perlopiù preoccupati di giustificare e tener fermo l'assunto di unidimensionalità, dall'altra i contributi degli altri scienziati sociali (sociologi politici, sociologi della cultura, psi-

2 È vero che il modello di Downs, e il suo assunto di unidimensionalità, hanno suscitato anche molte reazioni negative e molte critiche (Stokes 1963). Ma è altrettanto vero, come ebbe subito a rilevare Sartori, che tali critiche più che a «complicare» il modello, lavorando con spazi multidimensionali (Sartori 1965, 7), sembrano condurre all'abbandono del model approach in quanto tale.

3 Fra le alternative all'asse sinistra-destra prospettate nella letteratura è il caso di ricordare il continuum autoritario/democratico (Eysenck 1955), la coppia autorità/libertà (Bobbio 1992), il continuum laico-confessionale «cioè la dimensione religiosa» (Sartori 1965), la coppia governo opposizione (Weisberg 1974), il continuum materialismo/postmaterialismo (Inglehart 1977), la dimensione etnica (Sartori 1965). 
cologi sociali) strenuamente impegnati a metterlo in discussione attraverso l'adozione di modelli multidimensionali (solitamente a due o tre dimensioni).

Se si prova a fare un bilancio dei contributi degli uni e degli altri, due interpretazioni generali - ma si potrebbe anche dire: due atteggiamenti di fondo, due mentalita ${ }^{4}$ - si presentano abbastanza nitidamente. Per i primi il modello unidimensionale è, come tutti i modelli, una semplificazione della realtà. È inutile chiedersi se è vero o non lo è, perché - a rigore - esso è certamente (e ovviamente) falso'. Il problema è, semmai, quello di chiedersi se la semplificazione che esso introduce nella realtà sia accettabile oppure no, se la maggiore adeguatezza di un modello multidimensionale compensa la perdita di parsimonia implicita nella adozione di un modello più complesso. A queste domande Giovanni Sartori aveva risposto risolutamente no fin dai primi segnali di «fronda» contro il libro di Downs (Sartori 1965), e la scienza politica in Italia non sembra, da allora, essersi discostata troppo da quella linea di lavoro (Barnes 1971; Mannheimer e Sani 1987; Corbetta, Parisi e Schadee 1988). Quando gli oggetti da collocare nello spazio politico sono partiti e non gruppi sociali o altre entità più o meno connotate ${ }^{6}$, il massimo che i difensori dell'unidimensionalità sembrano disposti a concedere ai loro avversari è che l'unidimensionalità dello spazio politico sia «imperfetta». Sembra questa, ad esempio, la tesi prevalente fra gli studiosi dell'Istituto Cattaneo ${ }^{7}$, per i quali l'ordinamento dei partiti italiani lungo l'asse sinistra-destra «regge» lungo tutto il dopoguerra, purché si tenga conto della curvatura del continuum indotta dalla particolare posizione della Democrazia cristiana (Corbetta, Parisi e Schadee 1988, 367-68).

${ }^{4}$ Sull'attitudine drammatizzante dei sociologi, contrapposta all'attitudine normalizzante di altri scienziati sociali, vedi F. Rositi, richiamato in Ricolfi (1992a, 21-23).

s Sul punto vedi Hattie $(1985,147)$, che a sua volta non fa che riproporre una versione statistica del principio di Popper secondo cui di qualsiasi teoria scientifica, in quanto mera approssimazione alla «verità», si può tranquillamente predicare la falsità.

${ }^{6} \mathrm{Mi}$ riferisco, in particolare, all'impiego di «termometri dei sentimenti» su «stimoli» come il sindacato, l'Unione Sovietica, il clero etc. Vedi, ad esempio, Sani (1973, 1976), Marradi (1979).

7 Mentre questo articolo (scritto all'inizio del 1993) era all'esame della redazione della «Rivista Italiana di Scienza Politica» ho avuto notizia da Piergiorgio Corbetta di uno studio sulla struttura dello spazio elettorale in cui egli giunge a conclusioni consonanti con le mie, e un po' diverse da quelle classiche dell'Istituto Cattaneo. Un esame dei flussi elettorali 1987-1992 condotto con la tecnica dei modelli log-lineari sembra indicare l'impossibilità di mantenere l'ipotesi di unidimensionalità (Corbetta 1993). 


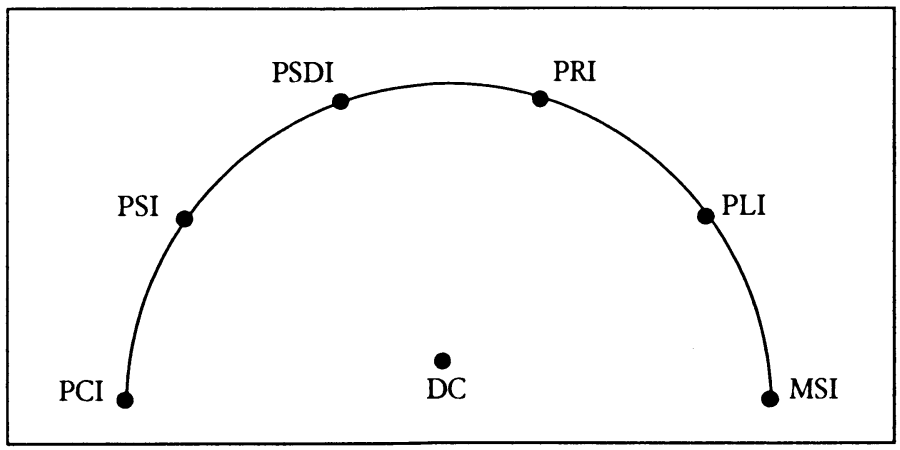

FIG. 1. Struttura dello spazio politico (tratto da Corbetta, Parisi e Schadee 1988, 367)

Alla tesi della «unidimensionalità imperfetta» altri studiosi hanno contrapposto quella che si potrebbe definire la tesi della «multidimensionalità emergente». Questa visione delle cose sembra basarsi su una sorta di valorizzazione della complessità, sull'idea che le semplificazioni introdotte dall'assunto di unidimensionalità rischino di nascondere proprio i fenomeni più interessanti e più nuovi che $\mathrm{i}$ movimenti collettivi degli ultimi vent'anni hanno introdotto nel sistema politico. Su questa linea, che non manca di seguaci anche all'estero ${ }^{8}$, si sono mossi in Italia sia gli studi sulle dimensioni dello spazio politico (Sani 1973 e 1976; Marradi 1979) sia alcune recenti analisi dello spazio elettorale vero e proprio (Biorcio e Lodi 1988; Biorcio 1991; Ricolfi 1988; 1993a; Sciolla e Ricolfi 1989). In queste analisi le relazioni fra i partiti vengono ricostruite mediante vari tipi di modelli spaziali associati ad altrettante tecniche di analisi dei dati ${ }^{9}$, con un numero di dimensioni o assi mai inferiore a due. Il significato di tali dimensioni cambia da caso a caso, e spesso non

${ }^{8}$ Si vedano Inglehart $(1977 ; 1990)$ e Raschke (1991) per la Germania, Stjernquist e Bjurulf (1970) per la Svezia, Hoskin e Swanson (1973) per la Colombia. Va detto, tuttavia, che in alcuni di questi lavori lo sforzo per catturare la complessità non si contrappone bensì si integra all'intento semplificante. Cogliendo un suggerimento dei matematici, che sogliono distinguere fra dimensionalità della rappresentazione e dimensionalità intrinseca di una figura, alcuni di questi autori prospettano una soluzione mista, che prevede rappresentazioni bidimensionali di fenomeni la cui dimensione intrinseca è 1 . Su questa interessante possibilità vedi Weisberg (1974).

${ }^{9}$ Le principali tecniche di analisi dei dati utilizzate sono l'analisi fattoriale, l'analisi delle corrispondenze, l'analisi discriminante, l'analisi in componenti principali. 
si limita ad integrare la classica contrapposizione fra sinistra e destra con nuove distinzioni, ma la fa scomparire del tutto sostituendola con nuove dimensioni il cui significato è irriducibile ad essa ${ }^{10}$.

Devo confessare che non solo le analisi del primo tipo ma anche quelle del secondo, ivi comprese alcune di cui sono responsabile io stesso ${ }^{11}$, non mi convincono affatto. Le prime perché le condizioni di accettazione del modello unidimensionale sono troppo deboli. Le seconde perché basate su una mancata problematizzazione del concetto di unidimensionalità e, conseguentemente, su un uso acritico di alcune tecniche di analisi dei dati. Lasciando da parte in questa sede le riserve sul primo tipo di approccio ${ }^{12}$ vorrei soffermarmi, in quel che segue, soprattutto sui limiti delle conclusioni tratte nell'ambito del secondo. Non mi riferisco tanto alla tesi della necessità di abbandonare il modello unidimensionale, che come si vedrà non è priva di riscontri empirici, quanto al modo di argomentarla e all'entità dello scostamento dal modello più semplice. In questo senso il lavoro che segue è anche un'autocritica e un invito a riaprire una discussione.

\section{Sul concetto di unidimensionalità}

$\mathrm{Ci}$ sono, nella letteratura metodologica, almeno sei o sette definizioni del concetto di unidimensionalità, almeno altrettante tecniche di analisi dei dati più o meno rigidamente connesse alle varie definizioni, e almeno quattro famiglie di dati empirici su cui l'assunto di unidimensionalità può essere controllato.

10 È il caso di Biorcio (1991), Biorcio e Lodi (1988) e Ricolfi (1988).

"Mi riferisco, in particolare, alle analisi fattoriali in Ricolfi (1988), Sciolla e Ricolfi (1989). Assai più difendibile mi sembra la soluzione bidimensionale ottenuta con l'analisi discriminante in Ricolfi (1993).

${ }_{12}$ Negli studi che lavorano su dati individuali (Barnes 1971; Sani 1974; Mannheimer e Sani 1987) l'elevata capacità esplicativa dell'autocollocazione sull'asse sinistra-destra non basta a provare l'unidimensionalità dello spazio politico; come notano giustamente Mannheimer e Sani; la stretta connessione fra autocollocazione e scelta politica lascia impregiudicata la direzione dei nessi causali (Mannheimer e Sani 1987, 117), e quindi non esclude che l'autocollocazione sia un costrutto derivato piuttosto che il supporto delle scelte elettorali.

Negli studi che lavorano sulle tavole dei flussi interpartitici (Corbetta, Parisi e Schadee 1988) l'ipotesi di multidimensionalità, più che risultare falsificata dai dati empirici, risulta incontrollabile per eccesso di parametri. 
Partendo da questi ultimi si può dire che la maggior parte delle analisi empiriche dello spazio elettorale utilizza una fra quattro grandi famiglie di dati, che diventano otto se si distingue fra dati statici (in cross-section) e dati dinamici (flussi, variazioni, serie temporali). Possiamo caratterizzarle così:

a) dati micro (survey), che per lo più registrano il grado di consenso di $\mathrm{N}$ soggetti a $\mathrm{K}$ partiti; tale grado di consenso può assumere la forma di un giudizio o atteggiamento verso ciascun partito (espresso da una variabile dicotomica, ordinale o cardinale), o quello di un vero e proprio ordinamento dei partiti in una sequenza che va dal più al meno «preferito»;

b) dati macro (ecologici), che per lo più registrano il grado di consenso (valutato attraverso il numero di voti ottenuti) di $\mathrm{N}$ unità territoriali a $\mathrm{K}$ partiti;

c) dati pico ${ }^{13}$ (infra-individuali), che per lo più registrano il grado di prossimità fra $\mathrm{K}$ partiti (stimoli) percepito da uno o più soggetti, oppure l'esito - in termini di preferenza - di tutti i confronti di coppia possibili fra i K partiti;

d) dati di gruppo, che perlopiù registrano il grado di accordo fra i comportamenti parlamentari dei rappresentanti di $\mathrm{K}$ partiti.

I politologi prediligono i dati dei tipi $a$ ) e $d$ ), i sociologi $\mathrm{i}$ dati del tipo a), gli psicologi sociali i dati del tipo $c$ ). Relativamente rare sono le analisi condotte con dati del tipo $b$ ). Esempi di analisi più o meno sofisticate dello spazio elettorale condotte con dati dei quattro tipi si possono trovare rispettivamente in Biorcio (1991), Biorcio e Lodi (1988), Tarrow (1967), Capecchi (1965), Stjernquist e Bjurulf (1970).

Distinguere fra i quattro tipi di dati è essenziale perché la loro natura condiziona fortemente il tipo di domande che ha senso porsi, la nozione di unidimensionalità che è concepibile adottare, nonché il genere di tecniche di analisi dei dati che è appropriato utilizzare. Se si lavora su una singola matrice di prossimità fra stimoli, ad esempio, non ha alcun senso utilizzare tecniche come l'unfolding o l'analisi fattoriale, che presuppongono dati two-way two-mode (Young e Hamer 1987). Così come non ha molto senso, se si lavora con risposte dicotomiche, pretendere di testare il rispetto della condizione di unidimensionalità nell'accezione di Black (single-peakedness).

${ }^{13}$ Seguo qui la terminologia di George Ainslie, che ha introdotto il termine «picoeconomics» per designare un programma di ricerca fondato su un livello di analisi infraindividuale (Ainslie 1985). 
Un discorso analogo potrebbe essere condotto sulle definizioni di unidimensionalità, nonché sulle tecniche di analisi dei dati. Per ora basterà notare che, se si considerano congiuntamente i tipi di dati, le definizioni di unidimensionalità e le tecniche di analisi dei dati, alla domanda «è unidimensionale lo spazio elettorale in Italia?» si può rispondere con almeno un centinaio di approcci diversi. Prima di entrare in medias res converrà quindi delimitare il campo per dare un senso preciso alla nostra domanda iniziale.

In questo lavoro opereremo esclusivamente su dati macro, aggregati a livello provinciale, e affronteremo il problema della dimensionalità dello spazio elettorale utilizzando esclusivamente l'analisi fattoriale. I nostri dati di partenza, in altre parole, sono costituiti da 95 casi (le province italian $\mathrm{e}^{14}$ ) e $\mathrm{K}$ variabili cardinali, tante quante sono le liste elettorali presenti in una determinata elezione ${ }^{15}$. Le tornate elettorali considerate sono quelle del $1953,1963,1972,1983,1992$. Ciò significa che qualsiasi conclusione da noi ottenuta dovrà essere interpretata in chiave macro, assumendo come unità di analisi non gli individui ma le unità territoriali.

$\mathrm{Ma}$ che significa che un certo risultato va interpretato in chiave macro? $\mathrm{E}$ in che senso, soprattutto, uno studio basato su dati territoriali può sfuggire alla «fallacia ecologica»?

A queste domande ho due tipi di risposte, una di tipo generale, l'altra legata al tipo di dati utilizzati in questo studio.

La prima risposta è che basta non dimenticare mai quel che si sta facendo. Se ci si ricorda che le unità sono territoriali basterà riferire le conclusioni al livello cui sono situate le premesse. Attribuire punteggi fattoriali alle unità ecologiche significa rappresentare la distribuzione territoriale del voto come effetto di una o più dimensioni che riassumono le tendenze di voto $o$, per dirla con Popper, le «propensioni» elettorali di ogni singola unità. Se il modello funziona non vuol dire che a decidere sono

14 Il numero delle province non è sempre il medesimo sia a causa delle variazioni intervenute nel dopoguerra nel numero delle stesse, sia a causa della necessità di escludere le province in cui una determinata lista non si presenta.

15 Anche il numero delle liste varia da elezione ad elezione, a causa delle scissioni e ricomposizioni dei partiti storici e, a partire dagli anni '70, a causa dell'apparizione di nuovi partiti. Per massimizzare la confrontabilità fra elezioni diverse le analisi sono state condotte sia su un elenco di variabili costituito da tutti e soli i partiti storici, sia - nelle ultime elezioni - su elenchi di variabili allargati alle principali liste nuove (Dp, Radicali, Verdi, Lega, Rete, Liste localiste). 
le unità ecologiche, né tantomeno che gli individui ragionano secondo le dimensioni evidenziate dall'analisi, ma solo che la distribuzione territoriale del voto si comporta come se fosse governata da una o più dimensioni latenti. E ovvio che si tratta di una astrazione e di una semplificazione, tutto sta a vedere se essa aiuta a capire come stanno le cose oppure no.

Né il fatto che le etichette attribuite a queste dimensioni (sinistra e destra, governo e opposizione etc.) possano echeggiare distinzioni e categorie tipicamente utilizzate a livello micro implica che anche l'interpretazione dei risultati si situi a questo livello. $\mathrm{Se}$, ad esempio, i partiti si dispongono lungo un asse fattoriale secondo l'ordinamento standard, da Rifondazione al Movimento sociale, non si vede perché non dovremmo etichettare l'asse stesso come sinistra-destra. Di nuovo, il problema non è di cambiare la terminologia, ma di essere consapevoli di quel che si sta facendo, nella fattispecie del fatto che le unità cui stiamo attribuendo delle proprietà sono unità territoriali e non individui.

Ma c'è anche una risposta più specifica, e al tempo stesso più tecnica, alle nostre domande iniziali. In estrema sintesi la formulerei così:

1) l'eventualità discussa da Robinson (che un'analisi micro rovesci i risultati di un'analisi macro) è limitata dalla struttura matematica del teorema di scomposizione della matrice varianze-covarianze $(\mathrm{T}=\mathrm{B}+\mathrm{W})$ che mostra che l'analisi macro (matrice $\mathrm{B}$ ) non è indipendente dall'analisi micro (matrice $\mathrm{T}$ ) ma ne costituisce una parte (Wilks 1962);

2) nel nostro caso è vero che l'analisi è condotta a livello macro, ma è anche vero che - contrariamente a quanto accadeva nel classico esempio di Robinson (1950) - non esiste una corrispondente analisi micro che potrebbe rovesciarne i risultati; poiché le dummies che danno origine alle nostre variabili aggregate sono fra loro incompatibili (il voto a un partito esclude il voto a tutti gli altri) la matrice $T$ è interamente occupata da correlazioni improprie, che assumono sempre valori negativi e i cui moduli dipendono esclusivamente dalla forza dei vari partiti ${ }^{16}$; detto più

${ }^{16}$ Ciò si deve all'ovvia circostanza che, a livello micro, le scelte elettorali non costituiscono un insieme di variabili logicamente indipendenti, ma corrispondono alle $\mathrm{K}$ modalità di un'unica politomia. Ne segue che, anche trasformandole in $\mathrm{K}$ dummies, ed eliminandone una per evitare ridondanze e collinearità, quel che si ottiene è una matrice varianze-covarianze artificiale, in cui la covarianza fra due variabili è semplicemente l'opposto del prodotto fra le rispettive probabilità (esempio: se il Pds ha una quota di 
esplicitamente: con dati di scelta o si fa un'analisi macro o non si fa alcuna analisi;

3) il nostro problema centrale non è individuare le «vere» dimensioni dello spazio elettorale, ma capire come variano nel tempo le dislocazioni reciproche dei vari partiti; questo significa che il lettore scettico può ignorare del tutto il sistema di riferimento e le relative etichette, e concentrare la propria attenzione sulle sole interdistanze fra i partiti.

Questo per quanto riguarda la scelta delle unità di analisi. Quanto alla definizione di unidimensionalità, adotteremo una variante di quella prevalente in ambito psicometrico (Hattie 1985). Un insieme di K variabili manifeste (gradi di consenso ai vari partiti, nel nostro caso) è unidimensionale se per spiegare $i$ dati bastano $K$ funzioni mono-argomentali $f_{k}(A)$, dove $A$ (che sta per «asse») è la variabile latente che governa le funzioni stesse.

In sé questa definizione è ancora insufficiente. Se la si prende alla lettera e non si pongono restrizioni sulla forma delle funzioni $f_{k}(A)$ è sempre possibile costruire $K$ funzioni abbastanza complesse (ossia dotate di abbastanza parametri) da permettere una ricostruzione perfetta della matrice dati in funzione di una sola variabile latente. Basterà ordinare $i$ casi in un modo qualsiasi e poi costruire $\mathrm{K}$ funzioni (polinomi di grado $\mathrm{N}-1$, ad esempio) tali da assicurare un adattamento perfetto. Occorre quindi o restringere l'ambito delle specificazioni funzionali possibili fissando il numero di parametri di ogni funzione, oppure - se non si vogliono imporre dei vincoli rigidi (di natura parametrica) alle funzioni - richiedere che queste rispettino almeno certi assunti di base. Il primo approccio è seguito tipicamente nelle tecniche parametriche (come il modello di Rasch), il secondo in quelle non parametriche (come il modello di Mokken).

Ma bisogna anche stare attenti a non esagerare con il rasoio di Ockham. Se si eccede nelle restrizioni può accadere di imporre condizioni assurde. Non mi riferisco tanto all'assunto di linearità delle funzioni quanto alla condizione apparentemente più ragionevole di monotonicità semplice, come nell'approc-

elettorato pari a 0.15 e la Dc pari a 0.20 la covarianza a livello micro fra voto al Pds e voto alla Dc è $-0.20 \times 0.15=-0.03$ ). Questo significa che - se si lavora sulle scelte effettive delle persone - l'analisi micro è logicamente priva di senso, e tecnicamente impossibile: è facile dimostrare che se tutte le correlazioni sono negative l'ipotesi di unidimensionalità è refutabile a priori (Ricolfi 1993b, cap. 6). 
cio classico ${ }^{17}$, o di monotonicità doppia, come in quello di Mokken ${ }^{18}$.

La prima condizione (monotonicità semplice) vieta che una o più funzioni siano crescenti per un certo tratto e decrescenti per un altro. La seconda condizione (monotonicità doppia) aggiunge che deve esistere una gerarchia tra le funzioni stesse: proprio perché rappresentano le probabilità di risposte «corrette» a una domanda $\mathrm{o}$ a un test, le funzioni che si riferiscono ad item più difficili devono assumere ovunque (ossia per tutti i valori di A) valori più bassi delle funzioni che si riferiscono ad item più facili. Graficamente questa condizione si può esprimere dicendo che le curve che rappresentano le varie funzioni non devono mai intersecarsi.

Questi criteri vanno benissimo quando si opera con batterie di item attitudinali di difficoltà crescente e riferiti alla medesima abilità latente, ma diventano assurdi non appena ciò che si desidera rilevare non è un'abilità ma una posizione politico-ideologica. In questi casi il minimo che si deve concedere alle funzioni $f_{k}(A)$ è di poter cambiare derivata almeno una volta, per consentire un'adeguata rappresentazione delle probabilità di scelta dei partiti di centro, il cui profilo è notoriamente prima crescente e poi decrescente (Barnes 1971, 134). Abbiamo quindi un'intera gerarchia di possibili funzioni monoargomentali:

(1) funzioni lineari (analisi fattoriale)

(2a) funzioni monotone crescenti a un parametro (Rasch 1960)

(2b) funzioni monotone crescenti a due parametri (Birnbaum 1968; Bock e Lieberman 1970)

(2c) funzioni monotone crescenti a tre parametri (Birnbaum 1968; Rogers 1984)

(3) funzioni «doppiamente monotone» (Mokken 1971), ma senza vincoli parametrici

${ }^{17}$ L'assunto di monotonicità semplice è implicito, ad esempio, nel ricorso all'analisi fattoriale. Esso ricorre inoltre in molti dei classici esempi di Lazarsfeld (Lazarsfeld 1960).

${ }_{18}$ In termini formali $\mathrm{i}$ due tipi di condizioni (monotonicità semplice e doppia) possono essere espressi così:

$$
\begin{aligned}
& \text { Monotonicità semplice: } \quad \mathrm{df}_{\mathrm{k}}(\mathrm{A}) / \mathrm{dA}>0 \\
& \text { Monotonicità doppia: } \quad \mathrm{df}_{k}(\mathrm{~A}) / \mathrm{dA}>0 \quad \mathrm{f}_{\mathrm{k}}(\mathrm{A})<\mathrm{f}_{\mathrm{i}}(\mathrm{A})
\end{aligned}
$$


(4) funzioni «a picco singolo», con al più un cambio di derivata (Lazarsfeld 1959)

(5) funzioni di forma qualsiasi (Lazarsfeld e Henry 1968; Etezadi 1981).

Graficamente i cinque tipi di funzioni si possono esemplificare così:

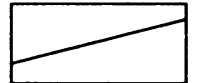

(1)

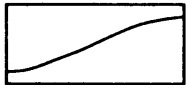

(2)

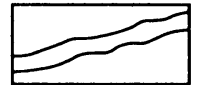

(3)

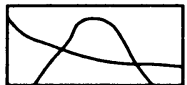

(4)

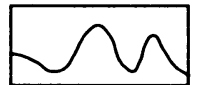

(5)

FIG. 2. Gerarchia di funzioni monoargomentali

Rispetto a questa gerarchia l'analisi fattoriale si situa al primo gradino, la nostra definizione di unidimensionalità si situa al quarto. In concreto questo vuol dire che la dimensionalità di una soluzione fattoriale non necessariamente coincide con la dimensionalità dei dati, almeno nell'accezione da noi scelta. Se come tecnica di analisi dei dati vogliamo o dobbiamo ${ }^{19}$ utilizzare un'analisi fattoriale i suoi risultati andranno interpretati con grande prudenza.

\section{Le trappole dell'analisi fattoriale}

$\mathrm{Ci}$ sono almeno due sensi in cui $\mathrm{i}$ risultati di un'analisi fattoriale possono essere fuorvianti.

Il primo è stato messo in rilievo quasi vent'anni fa da Shapcott e Wilson a proposito delle analisi dei bilanci-tempo (Shapcott e Wilson 1976) e vale per qualsiasi «sistema conservativo»: ogni qualvolta le variabili sottoposte ad analisi fattoriale sono a somma costante occorre fare $i$ conti con la singolarità della matrice di correlazione e, soprattutto, con la tendenza delle variabili stesse ad essere correlate fra loro in modo negativo (Ricolfi 1992b). Al primo problema si può porre rimedio esclu-

19 Il software che consente analisi fattoriali non lineari è a tutt'oggi una risorsa alquanto scarsa. Fra i pochi autori che hanno affrontato il problema delle specificazioni non lineari vedi Bartlett (1953), McDonald (1967; 1982), Wood et al. (1964), Lam (1980), Etezadi (1981), Etezadi e McDonald (1983). 
dendo una delle variabili, al secondo è impossibile ovviare senza trasformare le variabili. Se l'analisi fattoriale viene condotta sulle variabili originarie, sia pure ridotte a K-1, l'analisi fattoriale tenderà a sopravvalutare la dimensionalità dello spazio sottostante. Un fattore solo, infatti, non è mai in grado di riprodurre una matrice di correlazioni negative di ordine superiore a due (Ricolfi 1993b).

Il secondo senso in cui occorre guardarsi da un'interpretazione troppo diretta dei risultati di un'analisi fattoriale riguarda la forma delle relazioni fra le variabili manifeste e la variabile latente. Anche trascurando l'esistenza dei partiti di centro, ed ammettendo quindi che tutte le relazioni variabili-fattore siano monotone, la mera violazione della condizione di linearità basta ad innalzare la dimensionalità delle soluzioni ( $\mathrm{McD}$ Donald e $\mathrm{Ah}$ lawat 1974), con conseguente formazione di un certo numero di «fattori spuri» dovuti allo scostamento dalla linearità. Tale violazione è particolarmente probabile con i sistemi conservativi: il fatto che il grado di consenso ai vari partiti si comporti come una probabilità (come una quantità limitata inferiormente e superiormente) spinge le $f_{k}(A)$ ad assumere una forma non lineare, più o meno somigliante ad uno dei tanti modelli «a ogiva» proposti nella letteratura psicometrica ed econometrica ${ }^{20}$.

Questi problemi si accentuano, naturalmente, non appena si ammette - come appare inevitabile con i partiti di centro - che alcune delle $f_{k}(A)$ non dipendano monotonicamente dalla variabile latente $\mathrm{A}$. Se, ad esempio, lavoriamo con due partiti estremi e due partiti di centro la forma funzionale più semplice che si possa concepire è quella di due relazioni lineari e due relazioni paraboliche, o meglio di quattro relazioni paraboliche di cui due con i coefficienti dei termini quadratici vicini prossimi a zero:

$$
\begin{aligned}
& v_{1}=a_{1} A+0 A^{2} \\
& v_{2}=a_{2} A+b_{2} A^{2} \\
& v_{3}=a_{3} A+b_{3} A^{2} \\
& v_{4}=a_{4} A+0 A^{2}
\end{aligned}
$$

Per quanto schematico ${ }^{21}$, questo esempio mostra bene qual

${ }^{20}$ Fra le varie forme che il modello «a ogiva» assume ricordiamo le specificazioni logit, probit, burrit, gompit. Per una rassegna vedi Maddala (1983).

${ }^{21}$ Se le variabili manifeste sono probabilità o hanno un range limitato, la forma delle relazioni non lineari più che ad una parabola dovrebbe assomigliare ad una curva campanulare (una gaussiana, una Weibull o una Cauchy). 
è il problema che la non monotonicità solleva. Nonostante il processo generatore dei dati sia inequivocabilmente unidimensionale (tutto dipende dalla variabile latente A) l'analisi fattoriale tenderà a costruire due fattori, uno per cogliere il termine lineare $(A)$, l'altro per cogliere il termine quadratico $\left(\mathrm{A}^{2}\right)$.

Ciascuno di questi meccanismi (conservatività, funzioni non lineari o addirittura non monotone) conducono nella medesima direzione: l'analisi fattoriale tenderà a sopravvalutare la dimensionalità dello spazio sottostante. Per questo, come ho detto all'inizio, le analisi che hanno rivelato due o tre dimensioni dello spazio elettorale andrebbero riconsiderate con grande attenzione. Per poter dire che quelle analisi falsificano l'assunto di unidimensionalità occorrerebbe, come minimo, controllare che le dimensioni trovate non siano interdipendenti. In un caso, anzi, quello dei tre fattori trovati da Biorcio su un campione di elettori milanesi nel 1990, le prove portate a sostegno della conclusione possono essere completamente capovolte. Il fatto che l'analisi fattoriale mostri tre dimensioni e un'analisi delle corrispondenze ne mostri due si lascia interpretare, semmai, come un potente indizio di unidimensionalità. L'eccesso di dimensionalità dell'analisi fattoriale si può plausibilmente attribuire alla non monotonicità, mentre i due assi dell'analisi delle corrispondenze mostrano con grande chiarezza la presenza di una delle più classiche figure dell'unidimensionalità: il cosiddetto borseshoe (Kendall 1971), o Guttman effet (Benzécri 1973) o arch effect (Gauch 1982). Un'attenta ispezione del diagramma che descrive le relazioni spaziali fra i partiti mostra infatti che le loro proiezioni ortogonali si dispongono lungo una parabola che li ordina con ragionevole approssimazione da sinistra a destra.

Con questo, sia chiaro, non voglio passare direttamente sull'altra sponda, quella dei difensori ostinati dell'unidimensionalità, e tanto meno su quella dei multidimensionali pentiti. Quel che voglio dire è soltanto che per respingere l'ipotesi di unidimensionalità sulla base dei risultati di un'analisi fattoriale standard ci vogliono argomenti ben più solidi di quelli invocati dai suoi critici. Ma quali argomenti?

A mio parere per poter ragionevolmente affermare che un modello unidimensionale non rappresenta adeguatamente le relazioni fra i partiti occorre, per così dire, metterlo in grado di tenere conto di entrambi i problemi che abbiamo appena esposto. La matrice di correlazione non deve essere singolare, le variabili non devono contenere una tendenza alla correlazione in- 


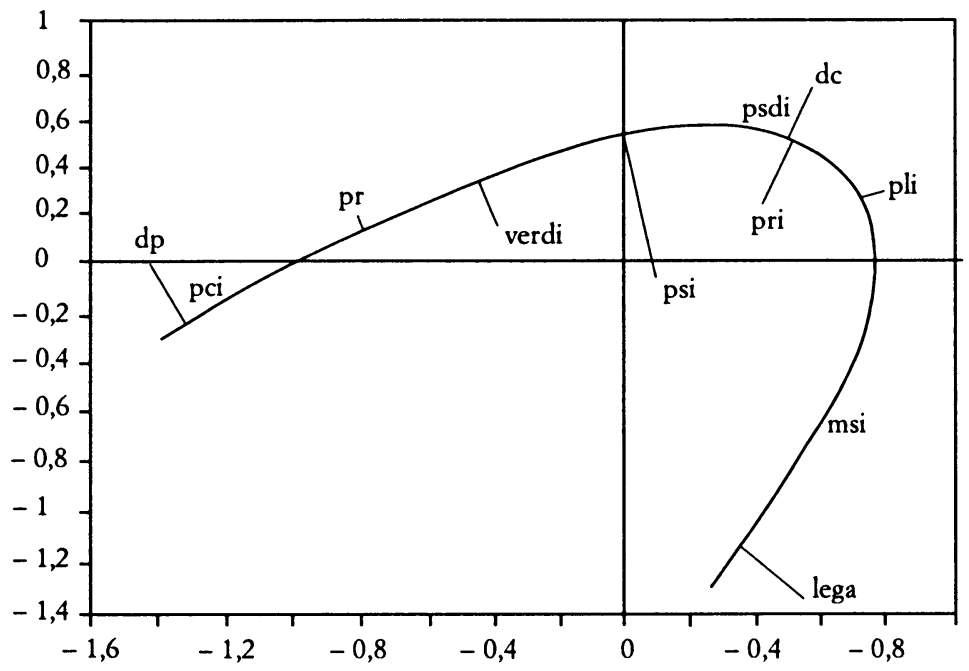

FIG. 3. Tipico Horseshoe (tratto da Biorcio 1991, 78)

Fonte: Sondaggio su elettori milanesi, 1990

versa, la specificazione delle relazioni fra variabili manifeste e variabili latenti deve consentire sia relazioni non lineari ma monotone («a ogiva») sia relazioni non lineari e non monotone («a picco singolo»). Queste condizioni sono soddisfatte da un'analisi fattoriale che, anziché operare sulle $\mathrm{K}$ variabili originarie, operi su K-1 trasformate logaritmiche delle variabili originarie stesse. Più esattamente l'eventualità di relazioni non lineari ma monotone può essere catturata da una trasformazione delle variabili manifeste che le sostituisca con i logaritmi delle relative «quote di scommessa» (probabilità di votare un partito diviso probabilità di non votarlo):

$\mathrm{v}_{\mathrm{k}}{ }^{\prime}=\log \left(\mathrm{v}_{\mathbf{k}} /\left(1-\mathrm{v}_{\mathrm{k}}\right)\right)$

L'eventualità di relazioni non lineari e non monotone (ma a picco singolo) può essere catturata da una trasformazione delle $\mathrm{K}$ variabili originarie che le sostituisca con $\mathrm{K}-1$ contrasti bilaterali indipendenti:

$\mathrm{v}_{\mathrm{kj}}{ }^{\prime}=\log \left(\mathrm{v}_{\mathrm{k}} / \mathrm{v}_{\mathrm{j}}\right)$ 
$\mathrm{Da}$ un punto di vista strettamente formale questa seconda specificazione corrisponde al cosiddetto strict utility model, proposto da Duncan Luce verso la metà degli anni cinquanta per formalizzare la relazione fra utilità e probabilità di scelta di $\mathrm{K}$ alternative indipendenti e mutuamente esclusive (Domenchic e McFadden 1975).

Con questi due insiemi di trasformazioni le condizioni statistiche per respingere l'ipotesi di unidimensionalità possono essere riformulate in modo più stringente. In quel che segue per giudicare della plausibilità dell'ipotesi di unidimensionalità ci baseremo sui seguenti indizi:

a) il rapporto fra secondo e primo autovalore della matrice di correlazione ${ }^{22}$;

b) la distorsion $\mathrm{e}^{23}$ associata alla soluzione unidimensionale nel caso del modello di Luce;

c) la distorsione associata alla soluzione unidimensionale nel caso del modello «a ogiva»;

d) il grado di interdipendenza ${ }^{24}$ fra primo e secondo fattore della soluzione a due fattori del modello «a ogiva».

Più precisamente l'ipotesi di unidimensionalità sarà considerata incompatibile con i dati se:

a) il secondo autovalore è almeno pari al $50 \%$ del primo;

b) la distorsione della soluzione unidimensionale nel caso del modello di Luce è superiore al $50 \%$;

c) anche la distorsione della soluzione unidimensionale nel caso del modello «a ogiva» è superiore al $50 \%$;

d) il grado di interdipendenza fra primo e secondo fattore della soluzione a 2 fattori del modello «a ogiva» è inferiore al $50 \%$.

La rappresentazione bidimensionale, a sua volta, sarà considerata una ragionevole ipotesi di lavoro solo se siamo in grado di respingere l'ipotesi di unidimensionalità (condizioni $a, b, c$, d) e, inoltre, la soluzione a due fattori del modello «a ogiva» presenta una distorsione inferiore al $50 \%$.

${ }^{22}$ Il criterio del rapporto fra primo e secondo autovalore è stato proposto da Hutten (1980) e Lord (1980).

${ }^{23}$ Come misura di distorsione utilizziamo, seguendo il package Spss, la percentuale di celle in cui il modulo dello scarto fra correlazioni effettive e correlazioni riprodotte supera 0.05 . Sul concetto di distorsione in analisi fattoriale vedi Ricolfi (1987).

${ }_{24}$ Il grado di interdipendenza è stato misurato effettuando due regressioni non lineari (con un termine lineare e un termine quadratico) fra i punteggi fattoriali dei due fattori e selezionando il coefficiente di correlazione multipla più elevato. 


\section{Una tendenza alla semplificazione?}

Può essere utile, per cominciare, dare uno sguardo d'insieme alle elezioni del dopoguerra, in modo da valutare se ha qualche fondamento la tesi - tipicamente difesa dai fautori della multidimensionalità - secondo cui la moltiplicazione delle issue e delle forze politiche intervenuta negli ultimi due decenni avrebbe aumentato il grado di complessità dello spazio politico.

Naturalmente, perché il confronto sia proponibile, occorre che le forze considerate nell'analisi siano sempre le stesse. Ciò sia per consentire un'analisi delle «migrazioni» dei vari partiti nello spazio elettorale sia, soprattutto, per non scambiare per un aumento di complessità dello spazio politico quella che è una semplice riduzione dei gradi di libertà del modello fattoriale. Aumentare il numero di partiti coinvolti, infatti, equivale ad aumentare la dimensione della matrice di correlazione, e con essa il numero di vincoli che una soluzione di una determinata dimensionalità dovrebbe rispettare ${ }^{25}$.

Per avere un'idea dell'evoluzione dello spazio elettorale nel dopoguerra abbiamo considerato i risultati di cinque tornate elettorali (elezioni politiche per la Camera dei Deputati), distanziate fra loro di circa un decennio: 1953, 1963, 1972, 1983, 1992. Come forze politiche abbiamo considerato i sette partiti sempre presenti nel quarantennio che va dal 1953 al 1992: Pci (Pds+Rc nel 1992), Psi, Psdi, Pri, Dc, Pli, Msi-Dn (compresi i Monarchici quando presenti).

I contrasti bilaterali utilizzati per mettere alla prova il modello di Luce sono stati costruiti fra partiti contigui, con l'Msi «contrastato» con le «Altre liste» ${ }^{26}$.

Prima di rendere esplicita la «semantica» delle nostre analisi, ossia il contenuto delle soluzioni trovate (posizioni dei partiti nello spazio elettorale), proviamo a soffermarci rapidamente

${ }^{25}$ Con 7 partiti e 2 fattori il modello fattoriale ha 21 vincoli, 13 parametri indipendenti da stimare e 8 gradi di libertà. Con 11 partiti i vincoli salgono a 55, i parametri indipendenti a 21 e i gradi di libertà a 34 .

26 La selezione delle province è stata effettuata in modo da assicurare che nessun contrasto fosse impossibile da calcolare a causa di un denominatore nullo. Ciò fa sì che le province sottoposte ad analisi non siano sempre esattamente le stesse. I risultati generali ottenuti, comunque, non variano ove l'analisi venga ripetuta operando su tutte e sole le province che non creano problemi di denominatore nullo. L'analisi fattoriale è stata condotta sempre con il metodo GLS (Generalize Least Squares), la rotazione con il metodo Varimax. 
sulla «sintassi», ossia sull'esito dei nostri cinque test (quattro contro l'ipotesi di unidimensionalità e uno a favore dell'ipotesi di bidimensionalità):

\begin{tabular}{|c|c|c|c|c|c|}
\hline & $\begin{array}{l}(\mathrm{a}) \\
\mathrm{l}_{2} / \mathrm{A}_{1}\end{array}$ & $\begin{array}{c}(b) \\
D_{\text {Luce }} \\
(\mathrm{H}=1)\end{array}$ & $\begin{array}{c}(c) \\
D_{\text {ogiva }} \\
(H \stackrel{1}{=})\end{array}$ & $\begin{array}{c}(\mathrm{d}) \\
\mathrm{I}\left(\mathrm{F}_{1}, \mathrm{~F}_{2}\right) \\
(\text { ogiva })\end{array}$ & $\begin{array}{c}(\mathrm{e}) \\
\mathrm{D}_{\text {ogiva }} \\
\left(\mathrm{H}^{2}\right)\end{array}$ \\
\hline 1953 & 79.4 & 71.0 & 52.0 & 42.4 & 4.0 \\
\hline 1963 & 89.4 & 66.0 & 76.0 & 21.9 & 47.0 \\
\hline 1972 & 73.9 & 66.0 & 61.0 & 4.5 & 42.0 \\
\hline 1983 & 72.1 & 52.0 & 80.0 & 6.3 & 42.0 \\
\hline 1992 & 63.7 & 61.0 & 85.0 & 49.0 & 23.0 \\
\hline
\end{tabular}

Come si vede in tutte le tornate elettorali i nostri dati non passano il test di unidimensionalità, ma superano quello di bidimensionalità. Sembrerebbe, a giudicare in modo meccanico questi risultati, che per rappresentare lo spazio elettorale nell'Italia del dopoguerra una dimensione non sia mai stata sufficiente, e tre dimensioni non siano mai state necessarie.

Personalmente non sarei così drastico, anche perché - come abbiamo cercato di sottolineare - raramente un'analisi fattoriale consente conclusioni definitive sulla dimensionalità. Quel che, invece, ci sembra possibile affermare con una certa sicurezza è che - contrariamente a quanto pensano molti osservatori - la complessità dello spazio elettorale non sembra affatto essere cresciuta negli ultimi trent'anni. Non solo, ma la maggior parte degli indizi contenuti nella tavola precedente suggerisce che la complessità dello spazio elettorale, che era relativamente bassa agli inizi degli anni cinquanta, abbia raggiunto il suo massimo storico verso la metà degli anni sessanta (elezioni del '63), per poi declinare nel trentennio che va dalla nascita del centro sinistra (elezioni del '63) alla dissoluzione dell'asse Dc-Psi (elezioni del 1992).

Se anzi si deve dire qual è l'anno in cui la tendenza alla semplificazione appare più pronunciata non vi è dubbio che questo è il 1992. In esso, infatti, due indicatori su quattro sono ai limiti della regione da noi convenzionalmente fissata per respingere l'ipotesi di unidimensionalità (colonne a e d), e i dati si trovano particolarmente «a loro agio» con il modello bidimensionale (distorsione inferiore al 25\%). Il sospetto, semmai, è che nel caso delle elezioni del 1992, così come in quelle del 1953, la forte dipendenza fra gli assi di una rappresentazione bidimen- 
sionale segnali una situazione di complessità intermedia fra il caso unidimensionale e quello bidimensionale ${ }^{27}$. $\grave{E}$ probabile, in altre parole, che un'analisi fattoriale non lineare, che consenta specificazioni non monotone possa assicurare un discreto adattamento anche ad una soluzione unidimensionale.

Questi risultati potranno sorprendere i sostenitori della tesi dell'aumento di complessità dello spazio elettorale, $\mathrm{ma}$ in fondo non sono poi così paradossali. Che l'aumento del numero di partiti potesse ridurre la dimensionalità dello spazio elettorale piuttosto che incrementarla è un'eventualità che Giovanni Sartori aveva già segnalato circa trent'anni fa, proprio a proposito dell'interpretazione «multidimensionale» di Stokes:

Mettiamola così: che le «percezioni di posizione» sono tanto più probabili e frequenti, quanto più aumenta il numero dei partiti. Per esteso: più sono $i$ partiti, tanto più la loro competizione acquista una disposizione spaziale, e tanto più si verifica in uno spazio lineare di qualche genere (questo dipenderà dalla «cultura» di ciascun paese). In non piccola parte ciò accade proprio perché a un numero crescente di partiti corrisponde un crescendo di ideologizzazione. $\mathrm{Ma}$ anche a prescindere dalla caratterizzazione ideologica, quando ci troviamo di fronte ai sistemi partitici «complessi», vale a dire quando l'elettore è posto di fronte a un sistema di otto o dieci partiti, una drastica semplificazione spaziale diventa una necessità. In conclusione: anche se crediamo che una semplificazione unidimensionale semplifichi troppo il caso elementare (quello dei sistemi bipartitici), tale semplificazione diviene tanto più realistica quanto più procediamo verso i casi complessi (Sartori 1965, 12).

Il quadro tracciato finora è volutamente astratto e puramente formale. Ci dice che la storia dello spazio elettorale nell'Italia del dopoguerra non è quella di una crescita più o meno regolare della dimensionalità ma è, semmai, quella di una parabola, di un'ascesa seguita da un lungo declino. Per passare dalla forma generale del processo ai suoi contenuti, per conoscere $i$ «personaggi» di questa storia, occorre capire come sono cambiate, in questi quaranta anni, le posizioni rispettive dei nostri sette partiti nello spazio elettorale.

Analizziamo dunque i cinque diagrammi (corrispondenti alle elezioni politiche considerate) che descrivono l'evoluzione dello spazio elettorale nel piano cartesiano individuato dai fattori della soluzione bidimensionale ${ }^{28}$.

${ }^{27}$ Può sembrare strano e improprio che qui si alluda ad una dimensionalità «intermedia» fra 1 e 2 . In realtà il concetto di dimensione non intera, frazionaria, o frattale è tutt'altro che privo di senso, almeno nella tradizione matematica (Mandelbrot 1987).

${ }^{28} \mathrm{Il}$ modello fattoriale utilizzato è quello in cui le variabili manifeste corrispondono ai logaritmi delle quote di scommessa di ciascun partito: $y^{\prime}=\log (y /(1-y))$. 


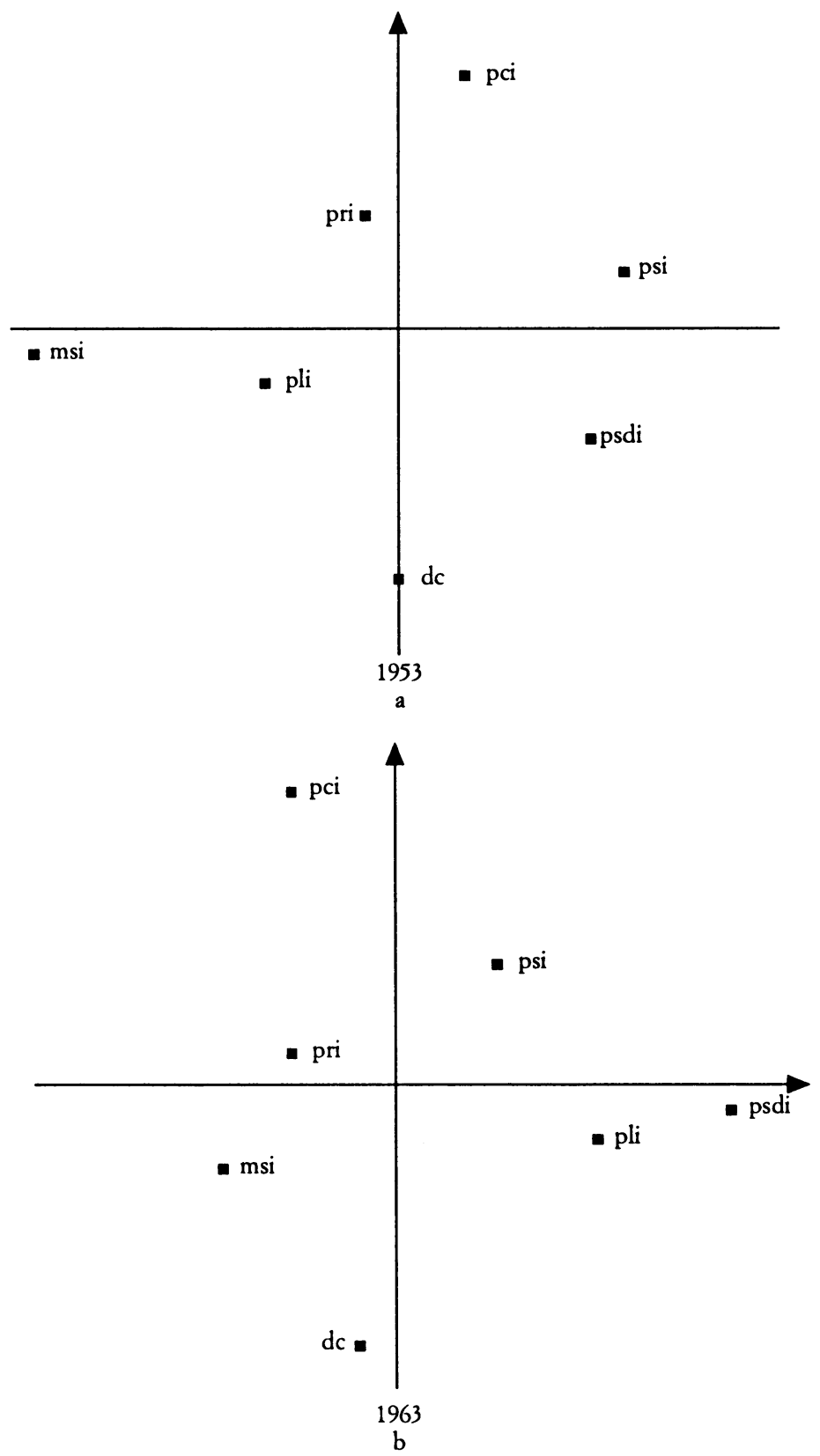

Fig. 4. Elezioni politiche del 1953 e del 1963 
Cominciamo dalle elezioni del 1953 e del 1963.

In entrambi i casi compare un asse (l'asse verticale) che contrappone le due grandi «chiese» del nostro sistema politico, quella cattolica (Dc) e quella marxista (Pci). Ma mentre nel 1953 l'altro asse (l'asse orizzontale) può essere plausibilmente interpretato come un continuum sinistra-destra che ordina gli altri partiti (la sequenza è esattamente quella «trovata» dall'Istituto Cattaneo lavorando sui flussi) nel caso delle elezioni del 1963 questo non è più possibile. L'asse che discrimina fra i partiti laici ci presenta infatti un ordinamento di ardua interpretazione: Msi, Pri, Psi, Pli, Psdi. È il caso di notare, inoltre, che in entrambe le configurazioni, ma soprattutto in quella del 1953, i partiti sono per lo più molto vicini ad un asse (quello che contribuiscono a definire) e, salvo il caso del Pri, decisamente lontani dall'altro asse. Questo significa che i dati, più che una situazione di multidimensionalità, rivelano una situazione di «unidimensionalità multipla»: gli oggetti rappresentati si dividono in più gruppi (due nel nostro caso), ciascuno dei quali appare scalabile in un proprio, particolare, spazio unidimensionale (Jacoby 1991, 35-37).

Nelle tornate elettorali successive $(1972,1983,1992)$ la struttura dello spazio politico cambia di nuovo, rivelando una sempre più accentuata tendenza al raggruppamento.

Nel 1972 si forma un primo raggruppamento che include tutti i partiti del centro laico (Psi, Psdi, Pri, Pli) e si contrappone ai tre partiti «ideologici» (Dc, Pci, Msi), che rappresentano le tre grandi tradizioni della storia del nostro paese: il cattolicesimo, il marxismo e il fascismo.

L'asse Dc-Pci è sempre presente (è anzi divenuto il primo asse della soluzione fattoriale), mentre l'altro asse coglie la contrapposizione fra movimento sociale e centro laico. Volendone fornire un'interpretazione lo si potrebbe ricondurre al continuum autoritario-democratico suggerito da Eysenck (1955), o alla coppia autorità/libertà, vista da Bobbio come alternativa (e ortogonale) rispetto alla coppia destra/sinistra (Bobbio 1992).

Nel 1983 il blocco dei partiti laici si divide. I liberali e i repubblicani da una parte, i socialisti e i socialdemocratici dall'altra.

I primi vanno ad occupare una regione dello spazio che come rivela un'analisi condotta con tutte le liste principali presenti alle elezioni del $1983^{29}$ - comprende anche Democrazia

29 Anche l'analisi allargata alle liste nuove ( $\mathrm{Dp}, \mathrm{Pr}$, Liste localiste) è stata condotta sui logaritmi delle quote di scommessa, secondo gli stessi criteri dell'analisi ristretta (a 7 partiti). Vedi nota 24. 


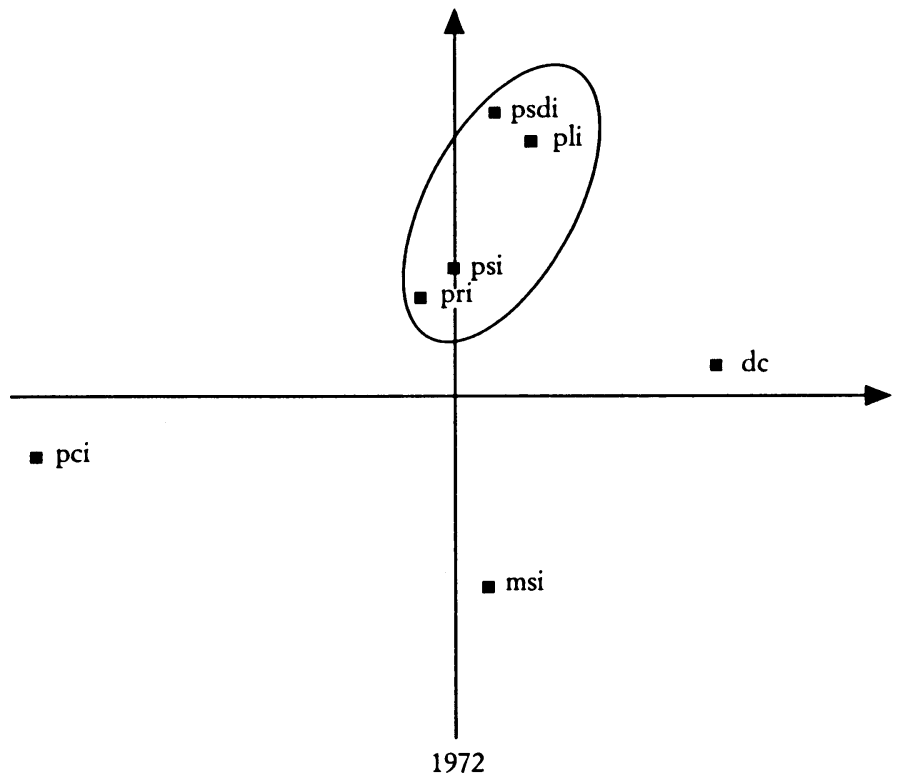

FIG. 5. Elezioni politiche del 1972

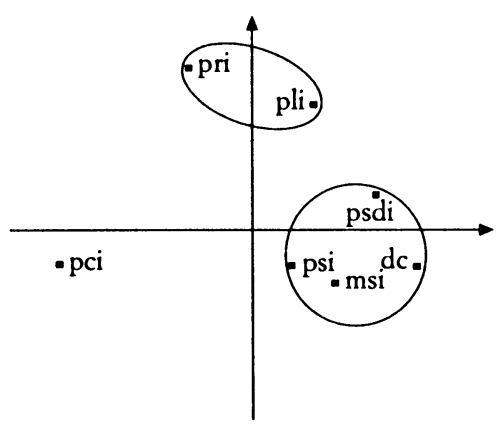

a

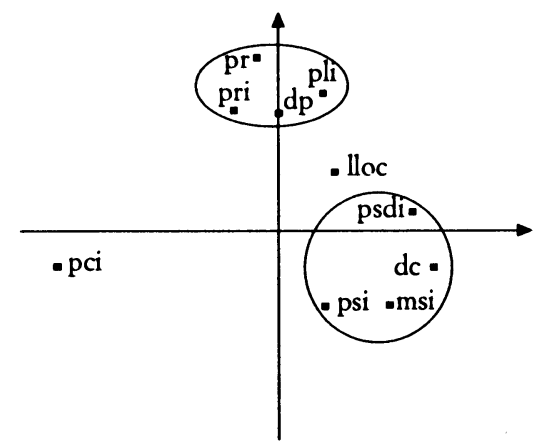

b

1983

FIG. 6. Elezioni politiche del 1983 
proletaria e il Partito radicale (fig. 6b). I secondi - socialisti e socialdemocratici - contribuiscono a formare un nuovo raggruppamento che comprende anche la Dc e il Msi. Volendo interpretare i due assi della soluzione fattoriale sembra inevitabile un richiamo al significato dei raggruppamenti in termini di insediamenti sociali e territoriali, soprattutto per quanto riguarda l'asse delle ascisse. Mentre l'asse delle ordinate, al cui polo positivo troviamo Pri, Pli, $\mathrm{Dp}$ e $\mathrm{Pr}$, si può interpretare sia in termini di insediamenti - come espressione delle tendenze di voto dei ceti medi metropolitani - sia in termini più direttamente politici - come espressione delle componenti più libertarie degli schieramenti referendari - per quanto riguarda l'asse delle ascisse l'unica interpretazione possibile sembra in termini di insediamenti territoriali: i partiti che gravitano intorno al polo positivo del primo asse (Msi, Dc, Psi, Psdi) altro non sono che $\mathrm{i}$ partiti del «blocco meridionale». Da questo punto di vista la novità che le elezioni del 1983 hanno immesso nello spazio elettorale sembra soprattutto quella di avere sostituito il vecchio asse Dc-Pci con una nuova contrapposizione, che accentua l'isolamento del Pci nella stessa misura in cui evidenzia la crescita di un «blocco assistenzial-conservatore», fondato sull'appoggio alla destra e ai partiti del «voto di scambio» (Cazzola 1988; Sciolla e Ricolfi 1989). Il significato di questo «blocco» è soprattutto territoriale, e segnala la progressiva meridionalizzazione della Dc e del Psi. Ma non è escluso che esso abbia anche un significato politico più profondo, quello di una sorta di «mutazione» dell'identità culturale del Psi. Non è tanto la contiguità fra Psi e Msi a colpire, quanto il fatto che questi due partiti, soprattutto nel diagramma allargato (fig. 6b), si collochino agli antipodi del cluster «libertario». Se l'asse delle ordinate viene letto, come nel 1972, come un continuum che coglie soprattutto la contrapposizione fra libertari e autoritari l'estrema contiguità fra Psi e Msi si colora anche di valenze politiche.

Nell'insieme le elezioni del passato sembrano indicare che solo in un anno, il 1963, la struttura dello spazio elettorale è abbastanza complessa da richiedere una rappresentazione multidimensionale in senso proprio ${ }^{30}$. Negli altri anni è la rappresentazione multidimensionale stessa a mostrarsi, in certo senso, più complessa del necessario. Prima del 1963 perché le posizioni

\footnotetext{
${ }^{30}$ Chiamo «rappresentazione multidimensionale in senso proprio» una rappresentazione in cui non vi sia né clustering né unidimensionalità multipla.
} 
reciproche dei partiti sembrano seguire un modello più semplice, quello della unidimensionalità multipla; dopo il 1963 perché le posizioni reciproche dei partiti sembrano seguire un modello ancora più semplice, quello del raggruppamento in pochi «poli» fondamentali ${ }^{31}$. Potremmo dire, con Ekman, che dopo il 1963 il sistema dei partiti perde progressivamente il suo carattere di «sistema dimensionale» per avvicinarsi sempre di più al modello ideale di un «sistema tipologico» (Ekman 1951a; 1951b).

Le elezioni del 1992 e l'eclissi dell'asse sinistra-destra

Nel 1992 le cose cambiano ancora, e la tendenza al raggruppamento si accentua.

Nel diagramma dello spazio elettorale non esistono più punti isolati (il Pci forma un cluster con il Pri), e anzi un'analisi condotta con tutte le liste principali, compresi quindi Verdi e Lega, mostra la comparsa di un terzo raggruppamento, in cui confluiscono tutte e tre le forze politiche di origine più recente - Lega, Verdi, $\mathrm{Pr}^{32}$ - indipendentemente dalla loro collocazione sull'asse sinistra-destra (fig. 6b). La struttura dello spazio politi-

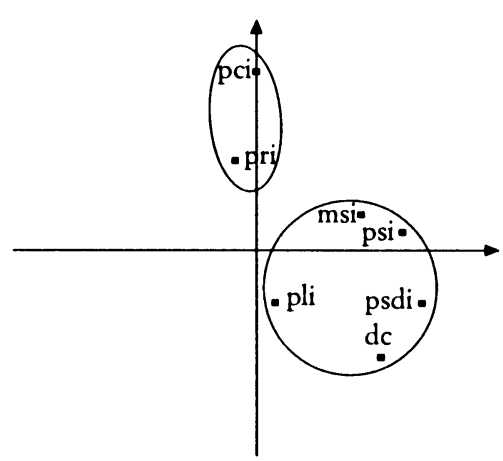

a

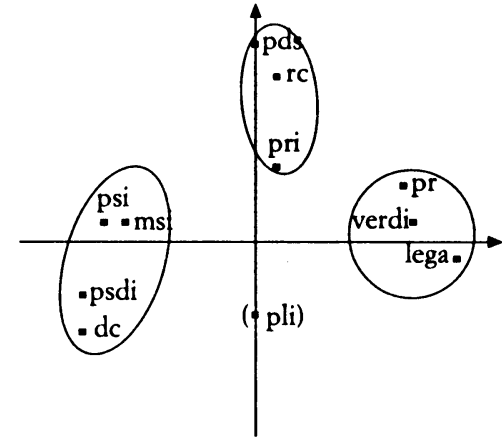

b

1992

FIG. 7. Elezioni politiche del 1992

${ }^{31}$ Per «poli» qui si intende regioni dello spazio elettorale occupate da un solo partito o da una «costellazione» di partiti molto prossimi fra loro.

${ }^{32}$ Con la sigla «Verdi» abbiamo sempre indicato l'insieme delle liste di ispirazione ambientalista. Con la sigla «Pr» abbiamo indicato il Partito radicale nel 1983 e la Lista Pannella nel 1992. 
co nel 1992 può dunque essere descritta come il risultato di un triplice evento: la dissoluzione del cluster «libertario» del 1983, la rottura dell'isolamento del Pci e la formazione di un terzo cluster, dovuta all'irruzione sulla scena politica di partiti completamente nuovi. Fra i tre eventi vi è una stretta connessione, perché ciascuno dei tre cluster in cui risulta suddiviso lo spazio elettorale nel 1992 beneficia della dissoluzione del cluster «libertario».

Il «blocco meridionale» tende ad assorbire nella propria orbita il Pli, una traiettoria che le vicende dell'ex ministro liberale De Lorenzo - coinvolto proprio a Napoli in un'inchiesta per voto di scambio - sembrano riassumere in modo emblematico. Nel 1992 il «blocco meridionale» non è altro che il quadripartito con l'aggiunta del Msi. In questo blocco colpisce l'estrema contiguità fra Psi e Msi, che era una delle novità del diagramma del 1983, e che non è detto sia interamente riconducibile al processo di «meridionalizzazione» del Psi. Quest'ultimo, infatti, era già iniziato negli anni sessanta (Gambetta e Ricolfi 1978), senza provocare effetti di questo tipo. Forse un pezzo della verità sta, semplicemente, nelle vignette di Forattini, che proprio nel decennio passato aveva preso a vestire Craxi con l'uniforme del Duce: in fondo gli anni ottanta sono anche gli anni del craxismo, un'epoca in cui la domanda di autorità si è ripresentata nelle forme più diverse - il decisionismo, la repubblica presidenziale, il «partito di Cossiga» - e ha finito per accomunare forze anche molto eterogenee fra loro (soprattutto socialisti, missini e liberali).

Nel blocco dei partiti nuovi - Verdi, Lega e Pr - confluisce il partito radicale, ossia il più libertario dei partiti del «blocco libertario». Sarebbe interessante capire se del medesimo blocco fa parte anche la Rete, ma il numero di province in cui essa era presente con proprie liste è troppo piccolo per consentirne una collocazione affidabile nello spazio elettorale ${ }^{33}$.

Il terzo ed ultimo blocco in cui si articola lo spazio elettorale - Pds, Rc, Pri - è il risultato del passaggio all'opposizione

${ }^{33}$ In realtà l'unica analisi che è stato possibile condurre con le liste della Rete un'analisi i cui risultati vanno presi con prudenza dato che coinvolge solo un terzo delle province - colloca il partito di Orlando vicino alla Dc. Questo risultato sembra riproporre una vecchia scoperta di Sidney Tarrow sui meccanismi elettorali del Mezzogiorno: gli antagonisti della Dc si sviluppano secondo la logica degli «anticorpi», tendendo a crescere dove la Dc è troppo forte, e a restare allo stato latente dove la Dc è debole (Tarrow 1967). 
dei repubblicani, ma anche della scissione del Pci-Pds e della conseguente confluenza di Dp in Rifondazione comunista. Quel che è estremamente significativo, in questo raggruppamento, non è la sua composizione interna quanto la regione dello spazio che esso va ad occupare. Se confrontiamo la disposizione dei partiti nel 1983 e nel 1992 possiamo renderci conto, infatti, che un modo di descrivere quel che è successo è di dire che nella regione «libertaria» del 1983 sono rimasti repubblicani e demoproletari (incorporati in Rc) e il posto di liberali e radicali è stato occupato dal vecchio Pci (Pds + la componente Pci di Rifondazione comunista). Nello stesso tempo la regione del vecchio Pci, che in tutte le soluzioni del dopoguerra si collocava agli antipodi della regione occupata dalla Dc, dando così luogo ad uno dei due assi dello spazio elettorale, risulta oggi occupata dai partiti nuovi.

Si può osservare, naturalmente, che il significato delle varie regioni cambia in funzione degli occupanti, e che una regione dello spazio che ha rinnovato i suoi occupanti non è più la stessa di prima. Si potrebbe notare, inoltre, che una diversa rotazione del sistema di riferimento permetterebbe di «ritrovare» l'opposizione fra Dc e Pci, sia pure complicata dall'annessione dei repubbblicani nell'orbita di quest'ultimo. Tutto questo è vero, ma non basta a cancellare un aspetto del diagramma del 1992 che è, per così dire, intrinseco e non dipendente dalle etichette che si possono attribuire alle varie regioni del piano: il fatto che, misurata in termini di varianza spiegata, la contrapposizione principale era ancora fra Dc e Pci nel 1983, ma è fra Dc e partiti nuovi nel $1992^{34}$. Questa circostanza si può rilevare osservando tre aspetti delle soluzioni a due fattori relative al 1983 e al 1992 (figg. 6b e 7b).

1. Nel 1983 il primo fattore (ossia quello che spiega la porzione più alta di varianza) oppone Pci e Dc; nel 1992 oppone Dc e partiti nuovi.

2. Nel 1983 il fattore su cui è «caricato» il Pci è bipolare (con la Dc all'altro polo), nel 1992 è «unipolare»; il loading dei liberali, infatti, è troppo basso per considerare bipolare il fattore ${ }^{35}$.

34 Nel 1983 il primo fattore rappresenta la contrapposizione fra Pci e Dc (modello «a ogiva»), nel 1992 rappresenta quella fra il blocco dello «status quo» e tre partiti nuovi: Pr, Verdi, Lega.

${ }_{35}$ Per questo la posizione dei liberali nel piano è stata indicata fra parentesi nella fig. $7 b$. 
3. Non esiste una rotazione del sistema di riferimento che permetta di «ritrovare» la contrapposizione fra Pci e Dc; il tentativo di costruire un asse che opponga Pci e Dc ha infatti come effetto quello di allontanarli dall'asse stesso (il che equivale ad indebolirne la caratterizzazione ${ }^{36}$ ).

Il terzo raggruppamento, che include i repubblicani e le due «anime» del vecchio $\mathrm{Pci}$, riunisce dunque le forze di quella che potrebbe essere definita l'«opposizione tradizionale» al blocco conservatore. Dove l'aggettivo «tradizionale» non si riferisce al fatto che le forze che lo compongono siano state tradizionalmente all'opposizione (l'uscita del Pri dalla maggioranza è piuttosto recente) ma al fatto che le forze che lo costituiscono sono sempre state presenti sulla scena politica del secondo dopoguerra.

La realtà che le elezioni del 1992 ci presentano si può leggere secondo due distinti registri. Il primo è quello di cercare di interpretare le due dimensioni che l'analisi fattoriale ha messo in luce, o comunque di trovare una rappresentazione bidimensionale che dia un senso generale ai nostri risultati. Su questa via si può anche dire, seguendo pedissequamente le scelte impersonali dell'algoritmo di rotazione (metodo Varimax), che le dimensioni dello spazio elettorale sono costituite da un asse (ascisse) che oppone i partiti nuovi al blocco conservatore e da un asse che raccoglie i partiti dell'opposizione tradizionale. $\mathrm{Ma}$ è probabilmente più proficuo decidere - in base a considerazioni puramente semantiche - di ruotare di circa 45 gradi in senso antiorario l'intero sistema di riferimento in modo da mettere in evidenza due nuove dimensioni, la cui interpretazione appare molto più semplice e naturale, se non altro perché facilmente riconducibile alle categorie del senso comune (fig. 8).

La prima di esse, che va da sud-ovest a nord-est, si può leggere come una dimensione di cambiamento: a un polo troviamo infatti quello che abbiamo definito il blocco conservatore, costituito dal Msi e dalle forze del quadripartito, all'altro polo troviamo tutti i partiti che si oppongono alla nomenklatura e sono portatori di un progetto di cambiamento o sul terreno dei contenuti o su quello delle regole del sistema politico o su entrambi. Se non fosse per la presenza del Msi nella stessa regione del

${ }^{36}$ I punti lontani da entrambi gli assi richiedono infatti interpretazioni miste, che si richiamano al significato di due assi anziché a quello di uno soltanto. 


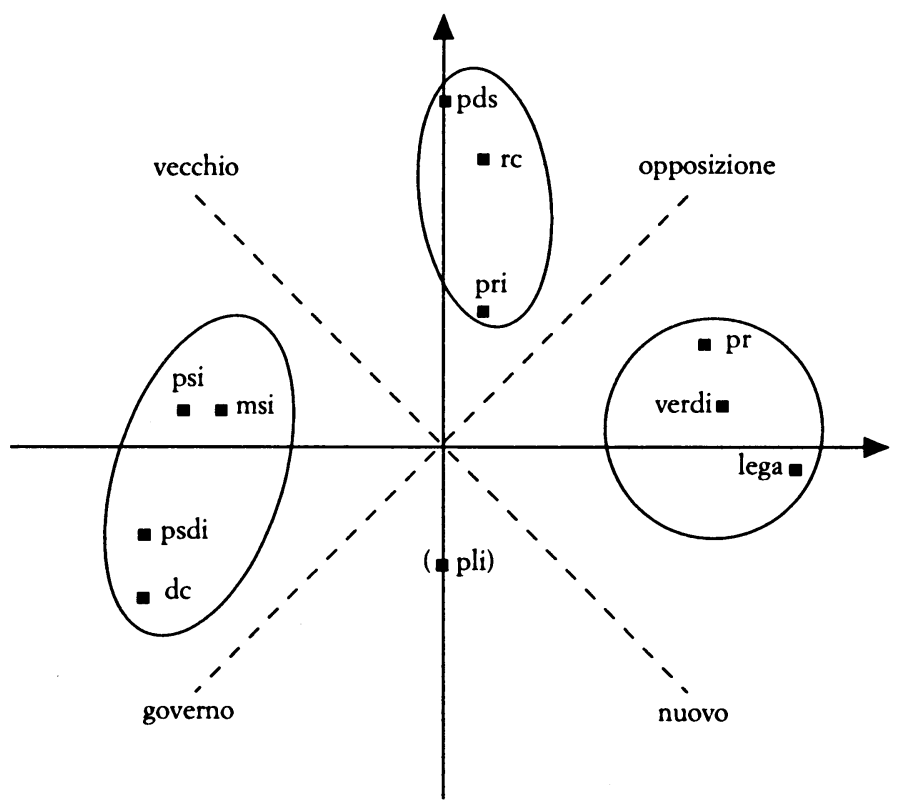

FIG. 8. Rotazione del sistema di riferimento

quadripartito, il polo del cambiamento si potrebbe anche definire come un polo di opposizione, che raccoglie tutte le forze che si oppongono alla maggioranza di quadripartito.

La seconda dimensione, che va da sud-est a nord-ovest, discrimina quasi esclusivamente ${ }^{37}$ fra i partiti del cambiamento, e lo $\mathrm{fa}-\mathrm{a}$ quanto pare - in base alla loro più o meno recente origine, o forse in base a una sorta di mediazione fra il criterio del grado di novità e quello della collocazione sull'asse destra-sinistra. Se ordiniamo $i$ partiti in base alle loro proiezioni ortogonali su questo secondo asse troviamo infatti: Lega, Verdi, Pr, Pri, Pds, Rc. Considerando che i tre partiti nuovi sono apparsi sulla scena politica proprio nell'ordine in cui li dispone il nostro asse novità/tradizione, e che il Pci - da cui Pds e Rc derivano - si porta dietro da sempre l'immagine di partito vecchio (Sciolla e Ricolfi 1989, 106-112) o, se si preferisce, di partito «che viene tenute.

${ }^{37}$ Le differenze fra i partiti tradizionali su questa dimensione sono piuttosto con- 
$\mathrm{da}$ lontano», questa interpretazione del secondo asse ci sembra tutto sommato accettabile.

Ma l'etichettamento degli assi della soluzione fattoriale è un esercizio relativamente astratto, e - nella fattispecie - forse anche un po' ingiustificato. Se lo spazio elettorale non rivela la struttura di un «sistema dimensionale» ma, semmai, quella di un «sistema tipologico», sembra molto più naturale e aderente alla natura dei dati etichettare direttamente i raggruppamenti che l'analisi ha messo in luce. Questo secondo registro permette di intravedere tre grandi poli elettorali: un blocco conservatore, o meglio favorevole allo «status quo», guidato dalla Dc, un blocco di «opposizione tradizionale» guidato dal Pds, e un blocco di «nuova opposizione» guidato dalla Lega. Questi tre blocchi, giudicati con le categorie ideologiche tradizionali, sono altamente disomogenei, perché contengono ciascuno, al proprio interno, una sinistra, un centro e - con l'eccezione dell'opposizione tradizionale - una destra. Se ci basiamo sulle autopercezioni degli elettorati dei vari partiti ${ }^{38}$ possiamo rappresentare la situazione così:

\begin{tabular}{llll}
\hline & Sinistra & Centro & Destra \\
\hline Status quo & Psi & Psdi $D c$ & Pli Msi \\
Opposizione tradizionale & Rc Pds & Pri & - \\
Nuova opposizione & Rete Pr & Verdi & Lega \\
\hline
\end{tabular}

E tuttavia non bisogna dimenticare che il risultato principale dell'analisi delle elezioni del 1992 è proprio l'ulteriore affievolimento dell'opposizione fra destra e sinistra come criterio di organizzazione dello spazio elettorale ${ }^{39}$. Si può pensare che questo sia un risultato transitorio, e che dopo la liquidazione di questa classe politica e il cambiamento delle regole elettorali l'asse sinistra-destra tornerà in primo piano. Si può ritenere che l'eclissi di quest'ultimo sia un artefatto della segmentazione geografica della società italiana, e che a livello individuale l'asse si-

${ }^{38} \mathrm{Ci}$ riferiamo alle autocollocazioni sull'asse sinistra-destra, quali risultano dalla maggior parte dei sondaggi. Per quanto riguarda le formazioni più recenti ci siamo basati sull'ultima indagine Iard (Ricolfi 1993).

${ }^{39} \mathrm{Ci}$ riferiamo, naturalmente, al solo livello ecologico. Nulla esclude che ad altri livelli l'asse sinistra-destra conservi intatta la sua capacità di organizzare lo spazio elettorale. 
nistra-destra mantenga intatta tutta la sua capacità di strutturazione degli orientamenti elettorali. Resta il fatto, comunque, che a questo livello di analisi (macro) le distinzioni importanti non sono fra sinistra, centro e destra (colonne della tabella) ma fra difensori dello status quo e fautori del cambiamento e, nell'ambito di questi ultimi, fra oppositori vecchi e nuovi.

\section{Tre poli, tre Italie: verso il paradosso di Arrow?}

Anche se i partiti-leader dei tre «poli» hanno una caratterizzazione abbastanza definita sull'asse sinistra-destra - il polo dello status-quo è guidato da un partito di centro, il polo dell'opposizione tradizionale è guidato da un partito di sinistra, il polo della nuova opposizione è guidato da un partito di destra $\mathrm{a}^{40}-$ lo stesso discorso non vale per l'insieme delle forze che costituiscono i tre poli. Per caratterizzare i tre poli occorre quindi rivolgersi ad altri criteri. Trattandosi di un'analisi condotta su dati ecologici un modo molto naturale di cogliere le differenze fra $\mathrm{i}$ tre poli è quello di valutare se le differenze stesse abbiano o meno un contenuto territoriale interpretabile ${ }^{41}$. A questo scopo abbiamo stimato i punteggi fattoriali di tutte le unità ecologiche sulle due dimensioni messe in evidenza dall'analisi ${ }^{42}$ e, a partire dai punteggi stessi, abbiamo costruito tre cluster ${ }^{43}$. Il risultato è riportato nella cartina riportata nella fig. 9 .

Se si considera che nessun vincolo di contiguità è stato imposto alle soluzioni il risultato appare di una nitidezza impressionante. La cluster analysis ripropone la suddivisione puramente geografica dell'Italia in un Nord, un Centro e un Sud o, per riprendere la terminologia di un recente e similare risultato ottenuto da Sani, la suddivisione dell'Italia in Padania, Etruria e Mezzogiorno (Sani 1992).

\section{destra.}

40 Il concetto di destra è qui usato in senso lato, e non come sinonimo di estrema

${ }^{41}$ Devo questo suggerimento a Roberto Cartocci, che mi ha segnalato la potenziale convergenza fra i miei risultati e quelli di Sani (1992).

42 Il calcolo dei punteggi è stato effettuato a partire dal modello «a ogiva», con il metodo della regressione. Le unità territoriali considerate sono state 93 su 95 , perché nell'analisi fattoriale eravamo stati costretti ad escludere le province di Aosta e Trieste.

${ }^{43}$ Il metodo utilizzato per il raggruppamento è la minimizzazione della traccia di W (matrice varianze-covarianze Within); il package utilizzato è Helga. La varianza spiegata dalla partizione in tre gruppi è stata pari al $41.8 \%$. 


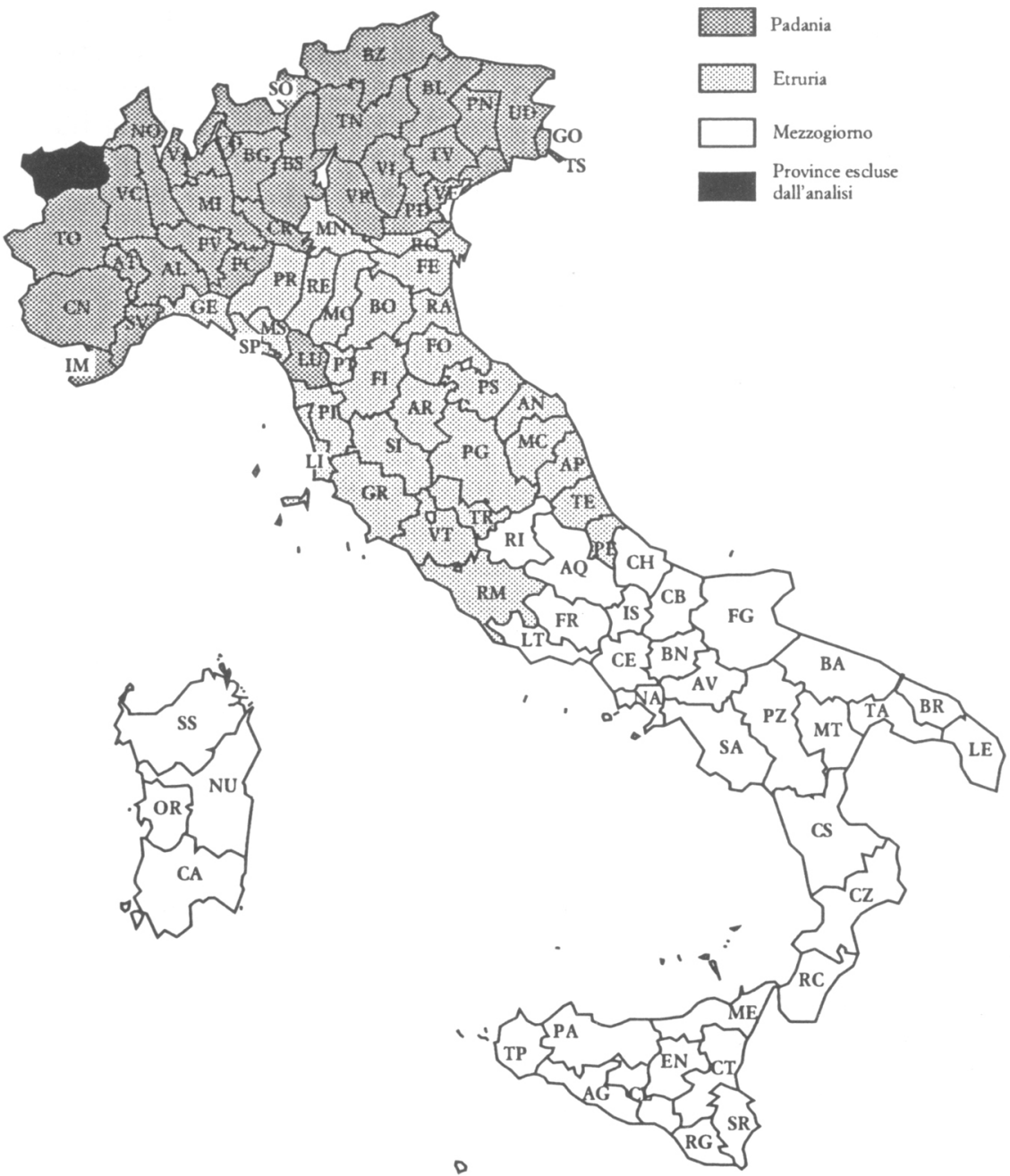

FIG. 9. Partizione dell'Italia basata sui punteggi fattoriali 


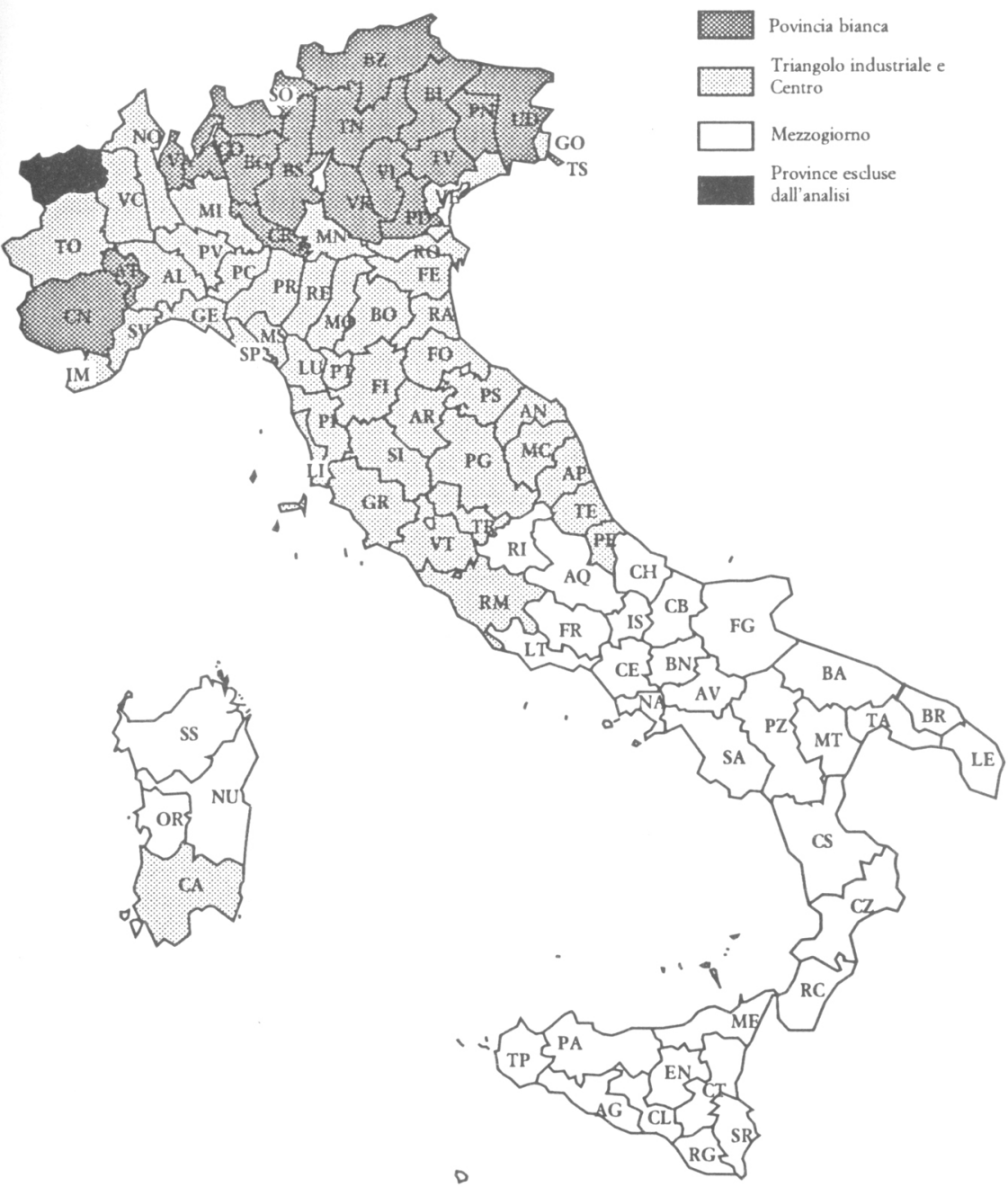

FIG. 10. Partizione dell'Italia basata sui consensi ai partiti 
I risultati differiscono un po' se, anziché lavorare sui punteggi fattoriali, lavoriamo direttamente sulle 11 variabili da cui i punteggi fattoriali sono ricavati ${ }^{44}$ (fig. 10).

In tal caso scompare l'enclave di Lucca e l'Italia centro-settentrionale, anziché scindersi in un Nord e in un Centro, tende a suddividersi in un'area che si potrebbe definire di «provincia bianca», che comprende il Triveneto e parte della Lombardia, e in un'area di tipo «metropolitano-secolarizzato», che include il triangolo industriale, le «regioni rosse», le Marche e parte del Lazio e dell'Abruzzo ${ }^{45}$.

$\grave{E}$ immediato rendersi conto che le tre aree, sia nella prima sia nella seconda partizione, corrispondono anche alle zone di massimo insediamento dei partiti che guidano i tre poli elettorali: la Lega al Nord, il Pds al Centro, la Dc al Sud. Ma queste corrispondenze valgono anche per i partiti satelliti?

Per avere una risposta basta calcolare le quote di consenso ottenute dalle diverse liste nelle tre aree. Ecco i risultati, separatamente per le due tripartizioni dell'Italia (marcata in grassetto la zona di massimo insediamento).

\begin{tabular}{lrrrrrr}
\hline & \multicolumn{3}{c}{ Punteggi fattoriali } & \multicolumn{3}{c}{ Variabili originarie } \\
& Sud & Centro & Nord & Sud & Centro & Nord \\
\hline Msi & $\mathbf{6 . 4}$ & 5.0 & 3.9 & 6.4 & 4.9 & 3.4 \\
Dc & 40.6 & 24.6 & 26.9 & 40.9 & 24.3 & 29.4 \\
Psi & 16.0 & 12.6 & 11.7 & 15.9 & 12.7 & 11.0 \\
Psdi & 4.7 & 2.0 & 2.0 & 4.7 & 2.1 & 1.8 \\
Pli & $\mathbf{2 . 8}$ & 2.0 & 3.1 & 2.8 & 2.4 & 2.9 \\
& & & & & & \\
Pri & 3.8 & 4.9 & 3.5 & 3.7 & 4.6 & 3.2 \\
Pds & 13.3 & 27.1 & 10.7 & 13.3 & 23.1 & 8.2 \\
Rc & 4.8 & 8.3 & 4.8 & 4.7 & 7.9 & 3.4 \\
Pann. & 0.6 & 1.3 & 1.3 & 0.6 & 1.4 & 1.1 \\
& & & & & & \\
Rete & $\mathbf{2 . 2}$ & 0.8 & $\mathbf{1 . 8}$ & $\mathbf{2 . 3}$ & 1.0 & 1.9 \\
Verdi & 1.8 & 2.9 & 3.5 & 1.8 & 3.1 & 3.4 \\
Lega & 0.3 & 5.3 & $\mathbf{1 8 . 8}$ & 0.3 & 8.3 & $\mathbf{2 0 . 8}$ \\
Lloc & 0.5 & 0.4 & 4.7 & 0.4 & 0.8 & 6.8 \\
\hline
\end{tabular}

${ }^{44}$ Si tratta delle 11 variabili che rappresentano il peso elettorale (\% di consensi su 100 voti validi) dei sei partiti storici più Pds, Rc, Pr, Verdi, Lega. In questo caso il numero di osservazioni delle tre Italie è meno equilibrato, perché il Nord perde il Triangolo industriale, che viene accorpato al Centro. La varianza spiegata è pari al $42.8 \%$.

${ }_{45} \mathrm{Per}$ quanto riguarda queste due ultime regioni si direbbe che la zona centrale tenda ad incorporare le province costiere, e a lasciare al Mezzogiorno quelle più in. terne. 
Come si vede, con due eccezioni di lievissima entità, la corrispondenza è praticamente perfetta. Solo la Rete e il Pli (l'una esclusa dall'analisi fattoriale, l'altro caratterizzato da una comunalità molto bassa) appaiono «incerti» fra Nord e Sud, mentre tutti gli altri partiti (comprese le Liste localiste), rispettano pienamente le aspettative. I partiti dello «status quo» (S) hanno il loro insediamento modale nel Mezzogiorno, i partiti dell'«opposizione tradizionale» (T) lo hanno al centro e i partiti della «nuova opposizione» (N) lo hanno al Nord.

La nitidezza della tripartizione, e l'adeguatezza del modello «tipologico» è confermata, indirettamente, da un confronto fra il potere esplicativo della politomia associata alle tre Italie nella soluzione a tre cluster e il potere esplicativo delle prime due componenti principali. Nonostante il modello dimensionale sia intrinsecamente molto più potente del modello tipologico ${ }^{46}$ il guadagno di varianza spiegata associato al suo impiego non è particolarmente pronunciato (dal $43 \%$ al $57 \%$ ). Non solo, ma un'analisi discriminante condotta sulle tre Italie in funzione delle medesime variabili utilizzate nell'analisi fattoriale ripropone sostanzialmente le stesse aggregazioni dei partiti, nonché un potere discriminante perfetto: in tutte le province esaminate la conoscenza dei risultati elettorali basta per sapere di quale delle tre Italie la provincia fa parte ${ }^{47}$.

Il fatto che l'evoluzione del sistema politico sembri procedere verso la formazione di tre poli, fortemente caratterizzati in termini territoriali e dominati dagli unici tre partiti di cui al momento sia ragionevole prevedere una forza elettorale «a due cifre» ${ }^{48}$, non è privo di conseguenze rilevanti sul futuro della competizione partitica. Fra le conseguenze ovvie vi è, naturalmente, una crescita di importanza delle questioni che hanno un forte riferimento all'identità territoriale (la spesa pubblica nel Mezzogiorno, l'autonomia fiscale delle regioni, i problemi delle minoranze etniche).

${ }^{46}$ Le variabili latenti del modello dimensionale possono assumere $\mathrm{N}$ valori distinti, la variabile latente del modello tipologico ne può assumere solo $\mathrm{K}$, tanti quanti sono i tipi o cluster previsti. Sul rapporto fra modello dimensionale e modello tipologico vedi Ekman (1951a; 1951b).

47 Questo risultato è stato ottenuto con un'analisi discriminante condotta sulle 11 variabili corrispondenti alle 11 liste analizzate con l'analisi fattoriale. Con due funzioni discriminanti il modello ha permesso di classificare correttamente il $100 \%$ delle province.

48 Il Psi sembra infatti destinato a scendere sotto la soglia del $10 \%$, e la Lega ad oltrepassarla abbondantemente. 
Ma esistono anche conseguenze meno ovvie, prima fra tutte un salto di complessità nei problemi di ridisegno delle regole istituzionali ed elettorali. Se i poli sono compatti e ben separati $\mathrm{e}$, inoltre, lo spazio elettorale non è riducibile ad un continuum unidimensionale, allora il cambiamento delle regole del gioco si trova di fronte un dilemma fondamentale.

Una scelta che costringesse i partiti a coalizzarsi fra loro nell'ambito di tre schieramenti sarebbe estremamente aderente alla «geografia» del voto, ma creerebbe anche grandissimi elementi di instabilità. Se i tre schieramenti sono sufficientemente lontani l'uno dall'altro, e le quote di consensi da essi mobilitabili sono del medesimo ordine di grandezza (prossime al 30$35 \%$ ), allora dobbiamo attenderci:

a) che i tipi di coalizioni possibili siano tre (ST, SN, TN), e non due come in tutti i sistemi che hanno una sinistra un centro e una destra;

b) che la coalizione vincente sia determinata più dagli accordi di vertice fra gli schieramenti che dall'esito del voto;

c) che il governo del paese tenda a risolversi in una (schiacciante) alleanza geografica più che in una alleanza politica.

D'altro canto - e questo è l'altro corno del dilemma - una scelta in favore di un sistema bipolare, in cui i partiti sono costretti a coalizzarsi nell'ambito di due soli schieramenti, correrebbe il rischio opposto, quello di pagare un tributo troppo alto all'esigenza della stabilità: se i poli reali sono tre e il sistema elettorale ne impone due, allora la capacità del sistema politico di rappresentare le differenze dell'elettorato può risultare seriamente compromessa.

$\mathrm{Su}$ un piano molto astratto e generale questa difficoltà di trasformare preferenze individuali in scelte collettive non è altro che il nucleo logico del «paradosso del voto», scoperto da Borda e Condorcet alla fine del '700, e riscoperto da Arrow alla fine degli anni quaranta con il teorema di possibilità ${ }^{49}$. Se le alternative sono almeno tre, è possibile che esistano, simultaneamente, una maggioranza dei cittadini che preferisce $A$ a $B$, una maggioranza che preferisce $\mathrm{B}$ a $\mathrm{C}$, ma anche una maggioranza

49 La prima presentazione delle contraddizioni del sistema della maggioranza da parte di Arrow risale al dicembre 1948, con la lettura di un articolo alla società econometrica. I risultati di Arrow sul «teorema generale di possibilità», nonché le relative dimostrazioni, furono invece pubblicati solo nel 1951, con la prima edizione di Social Choice and Individual Values. 
che preferisce $\mathrm{C}$ ad $\mathrm{A}$. È chiaro che questa intransitività delle preferenze collettive mette in forse la regola principe della democrazia, la regola della maggioranza, e conferisce alle situazioni che consentono di scegliere fra tre alternative un potenziale di instabilità molto forte. Dobbiamo concluderne che è questo il tipo di situazione verso cui ci stiamo incamminando?

Per rispondere a questa domanda bisognerebbe studiare a fondo (analisi dei nuovi collegi, simulazioni del voto etc.) i nuovi meccanismi elettorali che il sistema politico italiano si è dato, ma soprattutto occorrerebbe sapere se le preferenze individuali fra $i$ tre schieramenti stanno evolvendo verso una configurazione «pericolosa», in cui il verificarsi del paradosso di Arrow diventi non solo possibile - perché le alternative sono tre - ma anche probabile - perché i profili di preferenza dei cittadini spingono in quella direzione. In mancanza di dati individuali tutto quel che possiamo fare è di cercare di formarci un'idea della situazione utilizzando tutti gli indizi di cui disponiamo. Ma quali sono, sul piano macro, i segnali che possono avvertirci del pericolo?

A mio parere essi sono due: il grado di simmetria fra le posizioni reciproche delle tre Italie, e la presenza - attuale o prevedibile - di profili di preferenza che violano la condizione di single-peakedness.

Per controllare il grado di simmetria basta calcolare le distanze, nel piano individuato dai punteggi fattoriali, dei baricentri delle tre Italie.

\begin{tabular}{lcccc}
\hline & Nord & Centro & Sud & Quota di pop. \\
\hline Nord & 0.00 & 2.09 & 2.24 & $(38.0)$ \\
Centro & & 0.00 & 2.03 & $(29.6)$ \\
Sud & & & 0.00 & $(33.4)$ \\
\hline
\end{tabular}

La matrice delle distanze non potrebbe fornire indicazioni più nette: le tre Italie hanno un peso demografico molto simile, e i loro baricentri si situano sui vertici di un triangolo equilatero quasi perfetto. Sia pure di poco, l'Italia più «eccentrica» è l'Italia settentrionale, mentre la coppia (relativamente) meno eterogenea è quella che aggrega il Sud al Centro. Si può proprio dire, da questo punto di vista, che le elezioni del 5 aprile 1992 hanno posto la «questione settentrionale». 
Per controllare il grado di coerenza dei profili di preferenza tutto quel che possiamo fare, con dati aggregati, è lavorare su un'astrazione come l'elettore medio di una determinata provincia, attribuendo alla provincia stessa l'ordine di preferenza implicito nel risultato aggregato. Con tre alternative $\mathrm{i}$ profili di preferenza possibili sono sei in tutto (tanti quante le permutazioni di tre oggetti), mentre quattro è il numero massimo di profili reciprocamente compatibili. Ecco i profili rilevati nelle nostre province.

$\begin{array}{lllr} & & & \% \\ \mathrm{~S} & \mathrm{~T} & \mathrm{~N} & 57.4 \\ \mathrm{~S} & \mathrm{~N} & \mathrm{~T} & 31.5 \\ \mathrm{~T} & \mathrm{~S} & \mathrm{~N} & 10.4 \\ \mathrm{~N} & \mathrm{~S} & \mathrm{~T} & 0.8\end{array}$

È facile controllare che i quattro profili precedenti sono reciprocamente compatibili e, una volta ordinati nella sequenza T-S-N, non violano la condizione di single-peakedness ${ }^{50}$.

Possiamo tirare un sospiro di sollievo e dichiararci al riparo dal paradosso di Arrow?

Non è detto, perché molto del risultato precedente non dipende da una intrinseca «regolarità» delle preferenze degli italiani ma dal carattere ancora embrionale del terzo schieramento, quello che aggrega i partiti nuovi intorno alla Lega. Attualmente, lasciando da parte le «altre liste» i rapporti di forza fra $\mathrm{i}$ tre schieramenti sono i seguenti ${ }^{51}$ :

$\begin{array}{ll}\text { Status quo } & 54 \% \\ \text { Opposizione tradizionale } & 26 \% \\ \text { Nuova opposizione } & 17 \%\end{array}$

Che cosa succederebbe se, come è facile prevedere, i partiti

so Per sincerarsene basta collocare le quattro sequenze lungo il continuum T-S-N, mostrando che l'ordinamento di preferenza associato ad ogni sequenza dipende dalla posizione lungo il continuum:

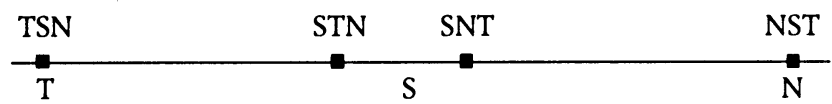

Come si vede le curve di utilità associabili alle 4 sequenze sono o monotone (sequenze estreme) o a picco centrale ma singolo (sequenze centrali).

${ }^{51}$ La somma delle tre percentuali non dà 100 perché dal calcolo abbiamo escluse le «Altre liste». 
dello status quo dovessero indebolirsi a favore dei partiti di opposizione, $o$ anche solo a favore dei nuovi partiti?

Un semplice esercizio di simulazione, che provi a redistribuire il $30 \%$ dei voti del primo schieramento verso gli altri due (un terzo all'opposizione tradizionale, due terzi a quella nuova), basta a mostrare che ciò basterebbe a fare apparire le due sequenze «proibite».

$\begin{array}{lllr} & & & \% \\ \mathrm{~S} & \mathrm{~T} & \mathrm{~N} & 43.1 \\ \mathrm{~S} & \mathrm{~N} & \mathrm{~T} & 7.5 \\ \mathrm{~T} & \mathrm{~S} & \mathrm{~N} & 18.4 \\ \mathrm{~T} & \mathrm{~N} & \mathrm{~S} & 1.3 \\ \mathrm{~N} & \mathrm{~S} & \mathrm{~T} & 25.6 \\ \mathrm{~N} & \mathrm{~T} & \mathrm{~S} & 4.1\end{array}$

Come si vede il nuovo insieme contiene tutte e sei le sequenze possibili, la cui compatibilità reciproca è esclusa a priori. $\grave{E}$ vero che questo non significa ancora, necessariamente, incappare nel paradosso del voto, che richiede anche proporzioni fra le varie condizioni tali da generare maggioranze intransitive e, soprattutto, richiede un sistema elettorale orientato in senso maggioritario, tale da spostare il cuore del processo decisionale dal singolo individuo alle unità territoriali $i^{52}$. Ma è anche vero che, con questa distribuzione territoriale dei voti e con l'abbandono del sistema proporzionale che la nuova legge elettorale ha sancito, la strada che porta ai paradossi della democrazia ${ }^{53}$ appare improvvisamente spianata.

$52 \mathrm{Se}$, ad esempio, le sei sequenze possibili vengono interpretate come politiche alternative, e le percentuali di consensi come grandezze proporzionali al numero di parlamentari eletti attraverso un meccanismo maggioritario (un parlamentare per ogni unità territoriale), diventa perfettamente concepibile una situazione del tipo:

$\begin{array}{llll}\mathrm{S} & \mathrm{T} & \mathrm{N} & 40.0 \\ \mathrm{~T} & \mathrm{~N} & \mathrm{~S} & 25.0 \\ \mathrm{~N} & \mathrm{~S} & \mathrm{~T} & 35.0\end{array}$

Con la struttura delle preferenze indicata la politica $\mathrm{S}$ prevale sulla politica $\mathrm{T}$ ( $75 \%$ di consensi), la politica $T$ sulla politica $\mathrm{N}(65 \%$ di consensi), ma quest'ultima, a sua volta, prevale sulla politica $\mathrm{S}(60 \%$ di consensi), violando così la condizione di transitività.

53 Il paradosso di Condorcet e il teorema di possibilità di Arrow sono solo alcuni esempi di paradossi della democrazia. Fra gli altri paradossi ricordiamo quelli di Ostrogorski e Anscombe (Bezembinder 1989), nonché il paradosso di Sen (Sen 1970). 
Riferimenti bibliografici

Ainslie, G. (1985), Beyond Microeconomics, in J. Elster (a cura di), The Multiple Self, Cambridge, Cambridge University Press.

Arrow, K.J. (1951), Social Choice and Individual Values, Cowles Commission Monograph 12, New York, John Wiley \& Sons; trad. it. Scelte sociali e valori individuali, Milano, Etas libri (trad. condotta sull'edizione americana del 1963).

Barnes, S.H. (1971), Modelli spaziali e l'identificazione partitica dell'elettore italiano, in «Rivista Italiana di Scienza Politica», I, pp. 123-43.

Bartlett, M.S. (1953), Factor Analysis in Psychology as a Statistician Sees $i t$, Uppsala Symposium on psychological factor Analysis, Uppsala, Almquist \& Wicksell, pp. 23-34.

Benzécri, J.P. (1973), L'Analyse des Données, Vol. 1: L'Analyse des Correspondances, Paris, Dunod.

Bezembinder, Th. (1989), Ostrogorski Paradox, in Encyclopedia of Statistical Sciences, Supplement Volume, New York, John Wiley \& Sons.

Biorcio, R. (1991), La Lega come attore politico: dal federalismo al populismo regionalista, in $\mathrm{R}$. Mannheimer (a cura di), La Lega Lombarda, Milano, Feltrinelli, pp. 34-82

Biorcio, R. e G. Lodi (a cura di) (1988), La sfida verde, Padova, Liviana.

Birnbaum, A. (1968), Some Latent Trait Models and their Use in Inferring an Examinee's Ability, in F.M. Lord e M.R. Novick, Statistical Theories of Mental Test Scores, Reading, Addison-Wesley, pp. 397-479.

Black, D. (1948a), On the Rationale of Group Decision Making, in «Journal of Political Economy», LVI, pp. 23-24.

- (1948b), The Decision of a Committee Using a Special Majority, in «Econometrica», XVI, pp. 245-61.

- (1948c), The Elasticity of Committee Decisions with an Altering Size of Majority, in «Econometrica», XVI, pp. 262-70.

Bobbio, N. (1992), Sinistra e destra. Un'alternativa che non è finita, in «La Stampa», 3 dicembre.

Bock, R.D. e M. Lieberman (1970), Fitting a Response Model for $n$ Dichotomously Scored Items, in «Psychometrika», XLVI, pp. 443-59.

Bosco, E. (a cura di) (1992), Ecologia e politica, Milano, Franco Angeli.

Borda, J.C. de (1781), Mémoire sur les élections au scrutin, in «Mémoires de l'Académie Royale des Sciences», pp. 657-65.

Bürklin, W.P. (1985), The German Greens. The Post-Industrial NonEstablished and the Party System, in «International Political Science Review», VI, pp. 463-81.

Capecchi, V. (1965), L'analisi delle preferenze politiche, in «Rassegna Italiana di Sociologia», II. 
Cazzola, F. (1988), Della corruzione, Bologna, Il Mulino.

Cofrancesco, D. (1984), Destra e sinistra, Verona, Bertani.

Condorcet, A. (1785), Essai sur l'application de l'analyse à la probabilité des décisions rendues à la pluralité des voix, Paris.

Coombs, C.H. (1950), Psychological Scaling without a Unit of Measurement, in «Psychological Review», LVII, pp. 145-58.

- (1952), A Theory of Psychological Scaling, in «Engineering Research Bulletin», XXXIV, Ann Arbor, University of Michigan Press.

- (1954), Social Choice and Strenght of Preference, in R.M. Thrall, C.H. Coombs e R.L. Davis (a cura di), Decision Processes, New York, John Wiley \& Sons.

Corbetta, P.G. (1993), La Lega e lo sfaldamento del sistema, in «Polis», VII, pp. 229-52.

Corbetta, P.G., A.M. Parisi e H.M.A. Schadee (1988), Elezioni in Italia, Bologna, Il Mulino.

Domencich, T. e D.M. McFadden (1975), Urban Travel Demand. A Behavioral Analysis, Amsterdam, North Holland.

Downs, A. (1957), An Economic Theory of Democracy, New York, Harper \& Row; trad. it. Teoria economica della democrazia, Bologna, Il Mulino, 1988.

Ekman, G. (1951a), On Typological and Dimensional Systems of Reference in Describing Personality, in «Acta Psychologica», VIII, pp. $1-24$.

- (1951b), On the Number and Definition of Dimensions in Kretschmer's and Sheldon's Constitutional Systems, in Essays in Psychology Dedicated to David Katz, Uppsala, Almquist and Wiksells, pp. 72-103.

Etezadi, J. (1981), A General Polynomial Model for Nonlinear Factor Analysis, in «Dissertation Abstract International», XLII.

Etezadi, J. e R.P. McDonald (1983), A Second Generation Nonlinear Factor Analysis, in «Psychometrika», XLII, pp. 315-42.

Eysenck, H.J. (1955), The Psychology of Politics, New York, Praeger.

Ferraresi, F. e G.E. Galeotti (1987), Destra/sinistra, in G. Zaccaria, Lessico della politica, Roma, Edizioni Lavoro.

Gambetta, D. e L. Ricolfi (1978), Il compromesso difficile. Forme di rappresentanza e rapporti di classe dal centro sinistra al compromesso storico, Torino, Rosenberg \& Sellier.

Gauch, H.G. (1982), Multivariate Analysis on Community Ecology, Cambridge, Cambridge University Press.

Giampaglia, G. (1990), Lo scaling unidimensionale nella ricerca sociale, Napoli, Liguori.

Greenacre, M.J. (1984), Theory and Applications of Correspondence Analysis, New York, Academic Press.

Hattie, J. (1985), Methodology Review: Assessing Unidimensionality of Tests and Items, in «Applied Psychological Measurement», IX, pp. 139-64. 
Hoskin, G. e G. Swanson (1973), Inter-Party Competition in Colombia, in «American Journal of Political Science», XVII, pp. 316-50.

Hosle, V. (1991), Philosophie der okologischen Krise, Munchen, Oscar Beck; trad. it. Filosofia della crisi ecologica, Torino, Einaudi (1992).

Hutten, L. (1980), Some Empirical Evidence for Latent Trait Model Selection, paper presentato al meeting annuale della «American Educational Research Association».

Inglehart, R. (1977), The Silent Revolution. Changing Values and Political Styles Among Western Publics, Princeton, Princeton University Press; trad. it. La rivoluzione silenziosa, Milano, Rizzoli, 1983.

- (1990), Culture Shift, Princeton, Princeton University Press; trad. it. Valori e cultura politica nella società industriale, Padova, Liviana, 1993.

Jacoby, W.G. (1991), Data Theory and Dimensional Analysis, London, Sage.

Kendall, D.G. (1971), Seriation from Abundance Matrices, in C.R. Hodson, D.G. Kendall e P. Tautu (a cura di), Mathematics in the Archaeological and Historical Sciences, Edinburgh, Edinburgh University Press.

Lam, R. (1980), An Empirical Study of the Unidimensionality of Ravens Progressive Matrices, Unpublished Master's Thesis, University of Toronto, Canada.

Lazarsfeld, P.F. (1959), Latent Structure Analysis, in S. Koch (a cura di), Psychology: A Study of a Science, New York, McGraw Hill.

- (1960), Latent Structure Analysis and Test Theory, in H. Gulliksen e S. Messick (a cura di), Psychological Scaling, New York, John Wiley \& Sons.

Lazarsfeld, P.F. e N.W. Henry (1968), Latent Structure Analysis, New York, Houghton-Mifflin.

Lord, F.M. (1980), Applications of Item Response Theory to Practical Testing Problems, New York, Erlbaum Associates.

McDonald, R.P. (1967), Nonlinear Factor Analysis, in «Psychometric Monographs», XV.

- (1982), Linear versus Nonlinear Models in Item Response Theory, in «Applied Psychological Measurement», VI, pp. 379-96.

McDonald-Ahlawat (1974), Difficulty Factors in Binary Data, in «British Journal of Mathematical and Statistical Psychology», XXVII, pp. 82-99.

Maddala, G.S. (1983), Limited-Dependent and Qualitative Variables in Econometrics, Cambridge, Cambridge University Press.

Mandelbrot, B.B. (1987), Gli oggetti frattali, Torino, Einaudi.

Mannheimer, R. (a cura di) (1991), La Lega Lombarda, Milano, Feltrinelli. Mannheimer, R. e G. Sani (1987), Il mercato elettorale, Bologna, Il Mulino.

Marradi, A. (1979), Dimensioni dello spazio politico in Italia, in «Rivista Italiana di Scienza Politica», IX, pp. 263-96. 
Mokken, R.J. (1971), A Theory and Procedure of Scale Analysis, The Hague, Mouton.

Rasch, G. (1960), Probabilistic Models for Some Intelligence and Attainment Tests, Copenhagen, Nielson \& Lydiche.

Raschke, J. (1991), Krise der Grünen. Bilanz und Neubeginn, Marburg.

Ricolfi, L. (1987), Sull'ambiguità dei risultati delle analisi fattoriali, in «Quaderni di Sociologia», XXXV.

- (1988), Il processo di secolarizzazione nell'Italia del dopoguerra: un profilo empirico, in «Rassegna Italiana di Sociologia», XXIX, pp. 37-87.

- (1992a), Crisi di teorie, crisi di indicatori, in «SIS-Bollettino», numero speciale, n. 26, pp. 12-29.

- (1992b), Sul rapporto di indicazione: l'interpretazione semantica $e$ l'interpretazione sintattica, in «Sociologia e Ricerca Sociale», 39.

- (1993a), Politica senza fede: l'estremismo di centro dei piccoli leghisti, in «Il Mulino», 345, pp. 53-69.

- (1993b), Tre variabili. Un'introduzione all'analisi multivariata, Milano, Franco Angeli.

Robinson, W.S. (1950), Ecological Correlations axd Behavior of Individuals, in «American Sociological Review», XV, pp. 351-57.

Rogers, H.J. (1984), Fit Statistics for Latent Trait Models, Unpublished master's thesis, University of New England, Armidale, Australia.

Sani, G. (1973), La strategia del PCI e l'elettorato italiano, in «Rivista Italiana di Scienza Politica», III, pp. 551-79.

- (1974), A Test of the Least Distance Model Of Voting Choice, Italy 1972, in «Comparative Political Studies», n. 2.

- (1976), La nuova immagine del PCI e l'elettorato italiano, in D.L.M. Blackmer e S. Tarrow (a cura di), Il comunismo in Italia e Francia, Milano, Etas.

- (1992), 1992: la destrutturazione del mercato elettorale, in «Rivista Italiana di Scienza Politica», XXII, pp. 539-65.

Sartori, G. (1965), Modelli spaziali di competizione tra partiti, in «Rassegna Italiana di Sociologia», II, pp. 7-29.

Sciolla, L. e L. Ricolfi (1989), Vent'anni dopo. Saggio su una generazione senza ricordi, Bologna, Il Mulino.

Sen, A. (1970), The Impossibility of a Paretian Liberal, in «Journal of Political Economy», LXXVIII, pp. 152-57.

Shapcott, M. e C. Wilson (1976), Correlations among Time Uses, in «Transactions of the Martin Center for Architectural and Urban Studies», 1.

Stjernquist, N. e B. Bjurulf (1970), Party Cobesion and Party Cooperation in the Swedish Parliament in 1964 and 1966, in «Scandinavian Political Studies», V.

Stokes, E. (1963), Spatial Models of Party Competition, in «American Political Science Review», 2, pp. 368-77. 
Tarrow, S.G. (1967), Peasant Communism in Southern Italy, New Haven, Yale University Press; trad. it. Partito comunista e contadini nel Mezzogiorno, Torino, Einaudi, 1972.

Thorndike, E.L. (1929), On the Fallacy of Imputing Correlations Found for Groups to the Individuals of Smaller Groups Composing Them, in «American Journal of Psychology», LII, pp. 122-24.

Weisberg, H.F. (1974), Dimensionland: An Excursions into Spaces, in «American Journal of Political Science», XVIII, pp. 743-76.

Wilks, S.S. (1962), Mathematical Statistics, New York, John Wiley \& Sons.

Wood, K.R., R.L. McCornack e L.T. Villone (1964), Non-linear Factor Analysis Program A-78A. TM-1764, Santa Monica, System Development Corp.

Young, F.W. e R.M. Hamer (a cura di) (1987), Multidimensional Scaling. History, Theory and Applications, Hillsdale, Lawrence Erlbaum Associates. 